CERN-TH.7292/94

GEF-TH-4/1994

\title{
Charm And BotTom Production: Theoretical Results Versus Experimental Data
}

\section{Stefano Frixione}

Dip. di Fisica, Università di Genova, and INFN, Sezione di Genova, Genoa, Italy

\section{Michelangelo L. Mangano ${ }^{1}$}

INFN, Scuola Normale Superiore and Dipartimento di Fisica, Pisa, Italy Paolo Nason ${ }^{2}$ and Giovanni Ridolfi ${ }^{3}$

CERN TH-Division, CH-1211 Geneva 23, Switzerland

\begin{abstract}
We present recent theoretical results in heavy-quark production, and we compare them with available experimental data. In particular, we consider the total cross sections, the $x_{F}$ and $p_{T}^{2}$ single-inclusive distributions, and results on heavy-quark pair correlations in both hadroproduction and photoproduction of charm and bottom hadrons.
\end{abstract}

CERN-TH.7292/94

June 1994

${ }^{1}$ Address after 1 February 1995: CERN, TH Division, Geneva, Switzerland

${ }^{2}$ On leave of absence from INFN, Sezione di Milano, Milan, Italy.

${ }^{3}$ On leave of absence from INFN, Sezione di Genova, Genoa, Italy. 


\section{Introduction}

Next-to-leading-order calculations of heavy-quark production cross sections have been developed in the last ten years by various authors (refs. [1-6] for hadroproduction and refs. [7-10] for photoproduction and electroproduction; some phenomenological applications are given in refs. [11,12]). In spite of the fact that a large amount of experimental data have been available for some time, the comparison of the full nextto-leading theoretical results and experiments has focused so far on few topics, such as the total charm cross sections and the transverse momentum spectrum of $b$ mesons. This was mostly due to the fact that it was considered unlikely that perturbative calculations could well describe charm production data (because of the smallness of the charm quark mass), and furthermore, because of limited experimental information on $b$ production. The lack of a full next-to-leading calculation of double-differential distributions was also a limiting factor in trying to understand the phenomenology of charm production in a more detailed fashion. This last problem has been overcome after the work in refs. [3,10], while in the mean time more data (both for charm and bottom at fixed target and in collider experiments) have become available. We therefore believe that it is now possible to undertake the comparison of theoretical results with data in a more systematic way.

In the present work we will deal with total, single-inclusive and double-differential cross sections for both charm and bottom production in hadron-hadron and photonhadron collisions. Our aim is twofold. On the one hand we will try to understand whether there are inconsistencies between perturbative QCD predictions and experimental results. This requires a thorough analysis of the theoretical uncertainties, in particular for the charm production case. As a second objective, we would like to see if, by a simple parametrization of the most important nonperturbative effects, we can give an adequate description of the observed phenomena. We therefore modified the calculations developed in refs. [3] and [10] in order to be able to take into account effects such as the primordial transverse momenta of the incoming partons and the hadronization phenomena.

We begin in section 2 with a discussion of total cross sections. In section 3 we examine the single-inclusive distributions of charm in both hadroproduction and photoproduction experiments, and the results on single-inclusive bottom production at hadron colliders. In section 4 we discuss the double-differential cross sections, and 
give our conclusions in section 5 .

\section{Total cross sections}

We report in table 1 some of the most recent experimental results for total charm hadroproduction cross sections. Almost all the measured cross sections are given with an $x_{F}>0$ cut. Theoretical studies [12] show that the ratio $\sigma / \sigma\left(x_{F}>0\right)$ is nearly independent of the heavy-quark mass and beam energy (at least for $m_{c}$ between 1.2 and $1.8 \mathrm{GeV}$, and the beam energy $E_{b}$ between 100 and $1000 \mathrm{GeV}$ ), and is approximately equal to 1.6 in pion-nucleon collisions and to 2 in proton-nucleon collisions.

Therefore, in order to obtain the total $D \bar{D}$ cross section from $\sigma\left(D / \bar{D}, x_{F}>0\right)$, we have to divide by 2 (to get the pair cross section from the single-inclusive one) and multiply by 1.6 (for pion) or 2 (for proton) to account for partial $x_{F}$ range coverage. Furthermore, the total $c \bar{c}$ cross section also receives contributions from $\Lambda_{c}$ and $D_{s}$ production. The corresponding cross sections are estimated to be (ref. [20] and references therein)

$$
\begin{aligned}
\frac{\sigma\left(D_{s}\right)}{\sigma\left(D^{0}+D^{+}\right)} & \simeq 0.2, \\
\frac{\sigma\left(\Lambda_{c}\right)}{\sigma\left(D^{0}+D^{+}\right)} & \simeq 0.3
\end{aligned}
$$

(here particle means also antiparticle). Therefore, to obtain $\sigma_{c \bar{c}}$ from the total cross section for $D \bar{D}$ production, we have to multiply by a factor of 1.5 . We stress that this value should be taken only as an indication. In fact, the results in eqs. (2.1) and (2.2) are affected by large errors. Furthermore, they are also based on the measurements of the E687 collaboration, that is, of a photoproduction experiment. Therefore, in using them to estimate the total cross section in charm hadroproduction, we neglect any correlation effect between the production and the hadronization phenomena. In this respect, it is interesting to notice that the NA14/2 collaboration [21] measures

$$
\begin{aligned}
& \frac{\sigma\left(D_{s}\right)}{\sigma\left(D^{0}+D^{+}\right)}=0.17 \pm 0.07 \pm 0.03 \\
& \frac{\sigma\left(\Lambda_{c}\right)}{\sigma\left(D^{0}+D^{+}\right)}=0.19 \pm 0.08,
\end{aligned}
$$




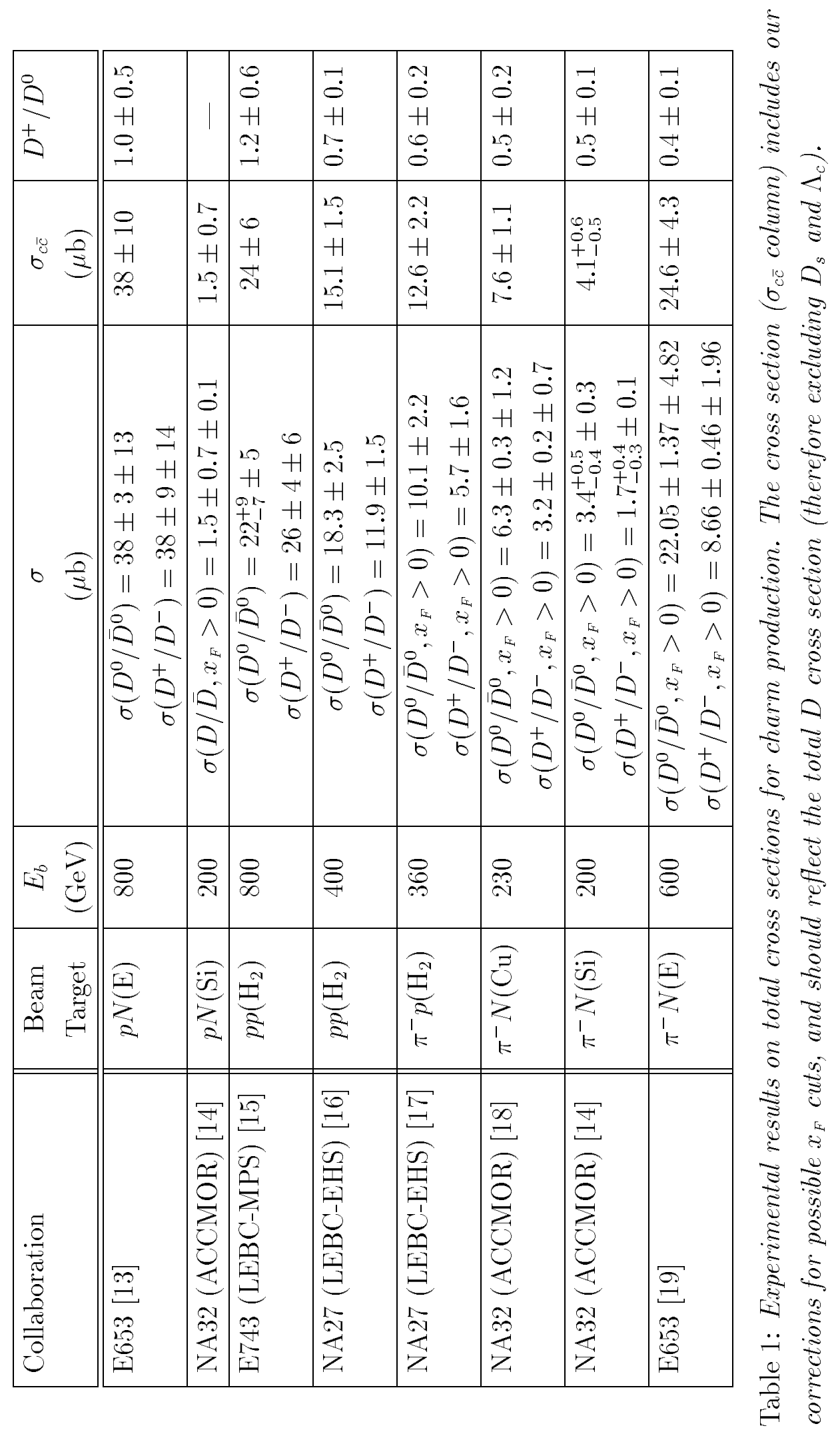


thus favouring a lower total $c \bar{c}$ cross section. The WA75 collaboration [20] does not present single-inclusive cross sections for $D$ mesons, but quotes directly

$$
\sigma_{c \bar{c}}=23.1 \pm 1.3_{-3.3}^{+4.0} \mu b
$$

assuming an $A^{0.87}$ dependence; this result was not inserted in table 1 . The E789 collaboration [22], using an $800 \mathrm{GeV}$ proton beam colliding on a Be or Au target, studied (in an experiment designed for beauty physics) neutral $D$-meson production near $x_{F}=0$ to investigate the $A$ dependence of the results. As a by-product, they obtain

$$
\sigma\left(D^{0} / \bar{D}^{0}\right)=17.7 \pm 0.9 \pm 3.4 \mu b
$$

An analogous measurement for charged $D$ cross section is not expected [23].

From table 1 we see that all the experimental collaborations but one report separately the total cross sections for charged and neutral $D$-meson production. In the last column of table 1 we have calculated the ratio $R$ between these two cross sections. For pion-nucleon collisions, the agreement among various collaborations is fairly good, and the errors on $R$ are moderate; the $D^{0}$ cross section is found to be twice as large as the $D^{+}$cross section. A simple model for estimating the charged-to-neutral $D$ cross section ratio is the following. One assumes that the $D$ cross section is suppressed by a factor of 3 with respect to the $D^{*}$ cross section, due to counting of polarization states, and then one uses the published values of $D^{*} \rightarrow D$ branching ratios to get the relative number of neutral and charged $D$. In this way, one gets roughly

$$
\frac{\sigma\left(D^{+}\right)}{\sigma\left(D^{0}\right)} \simeq 0.43 .
$$

This number is in reasonable agreement with the values reported in table 1 for $\pi N$ collisions. For $p N$ collisions, the same simple argument should hold. Experimental measurements seem instead to give comparable total cross sections for charged and neutral $D$. We do not find any reasonable explanation of this fact. We also estimated the charged-to-neutral $D$ cross section ratio using the Monte Carlo program HERWIG [24], which incorporates a cluster model for the charmed quark hadronization, phase space and number of polarization states to determine the $D / D^{*}$ ratio and available branching ratios for the decays. We found values fairly consistent with the estimate in eq. (2.5), for both pion and proton beams. On the other hand, the experimental 
data for the proton beam are much less clear than in the case of $\pi^{-} N$ collisions, the uncertainty on $R$ being quite large. The only $R$ value affected by a moderate uncertainty, the one reported in ref. [16], indicates a behaviour similar to that measured in $\pi^{-} N$ collisions.

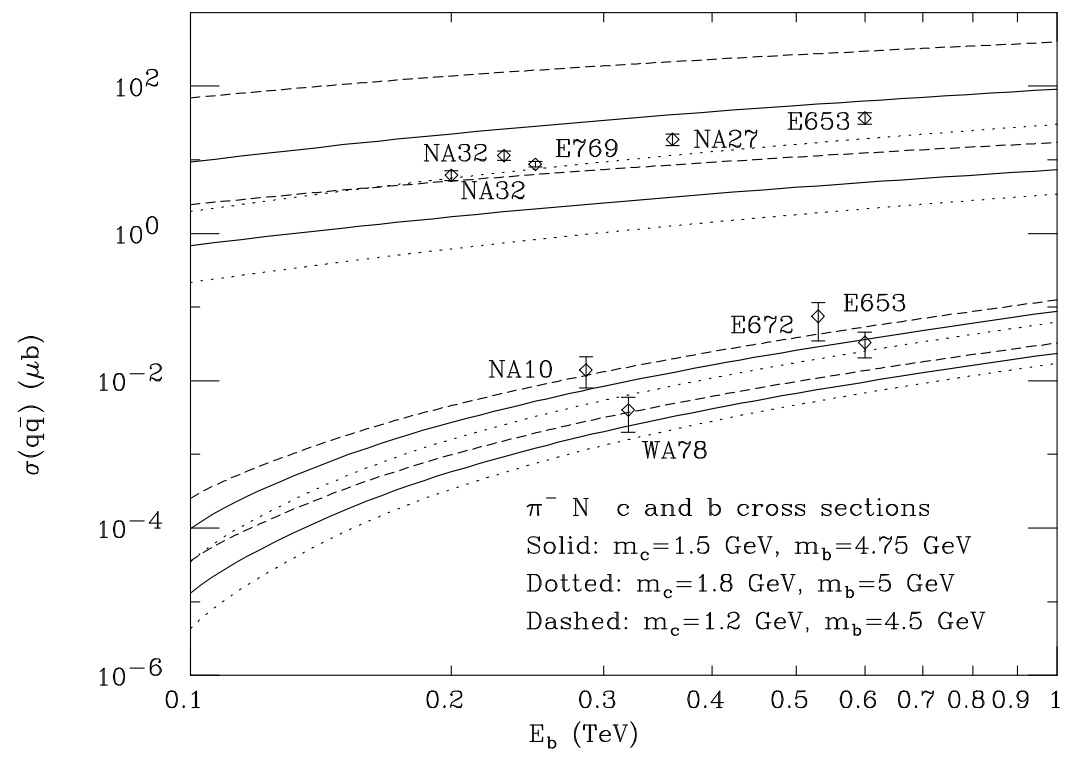

Figure 1: Cross sections for $b$ and $c$ production in $\pi N$ collisions versus experimental results.

In fig. 1 we plot the $c \bar{c}$ and $b \bar{b}$ cross sections, computed in QCD at next-to-leading order, as functions of the beam energy, for $\pi N$ collisions. The cross sections are calculated using the parton distribution set HMRSB [25] for the nucleon and the central set SMRS2 [26] for the pion. The default values of the charm and bottom masses are 1.5 and $4.75 \mathrm{GeV}$, respectively, and the default choices for the factorization scale $\mu_{F}$ and the renormalization scale $\mu_{R}$ are

$$
\mu_{R}=m_{c}, \quad \mu_{F}=2 m_{c}
$$

for charm and

$$
\mu_{R}=\mu_{F}=m_{b}
$$

for bottom. 
The bands in the figure are obtained as follows. We varied $\mu_{R}$ between half the central value and twice this value. The factorization scale $\mu_{F}$ was also varied between $m_{b} / 2$ and $2 m_{b}$ in the case of bottom, while it was kept fixed at $2 m_{c}$ in the case of charm. This is because available parametrizations of parton densities are usually given for $Q^{2}$ larger than 5 or $10 \mathrm{GeV}^{2}$. The bands represent the maximum variation of the cross sections in this parameter range. Due to the large uncertainties that one finds in charm production from renormalization scale and mass dependence alone, we found that it was not worth while to try to extend the available parton densities to smaller $Q^{2}$ values. The reader should therefore keep in mind that the bands shown in the figure are only an underestimate of the uncertainties involved in the computation of charm production cross sections. We verified that considering independent variations for the factorization and renormalization scale does not lead to a wider range in the bottom cross section for the energies shown in the figures. We also show the effect of varying $m_{c}$ between $1.2 \mathrm{GeV}$ and $1.8 \mathrm{GeV}$, and $m_{b}$ between 4.5 and $5 \mathrm{GeV}$.

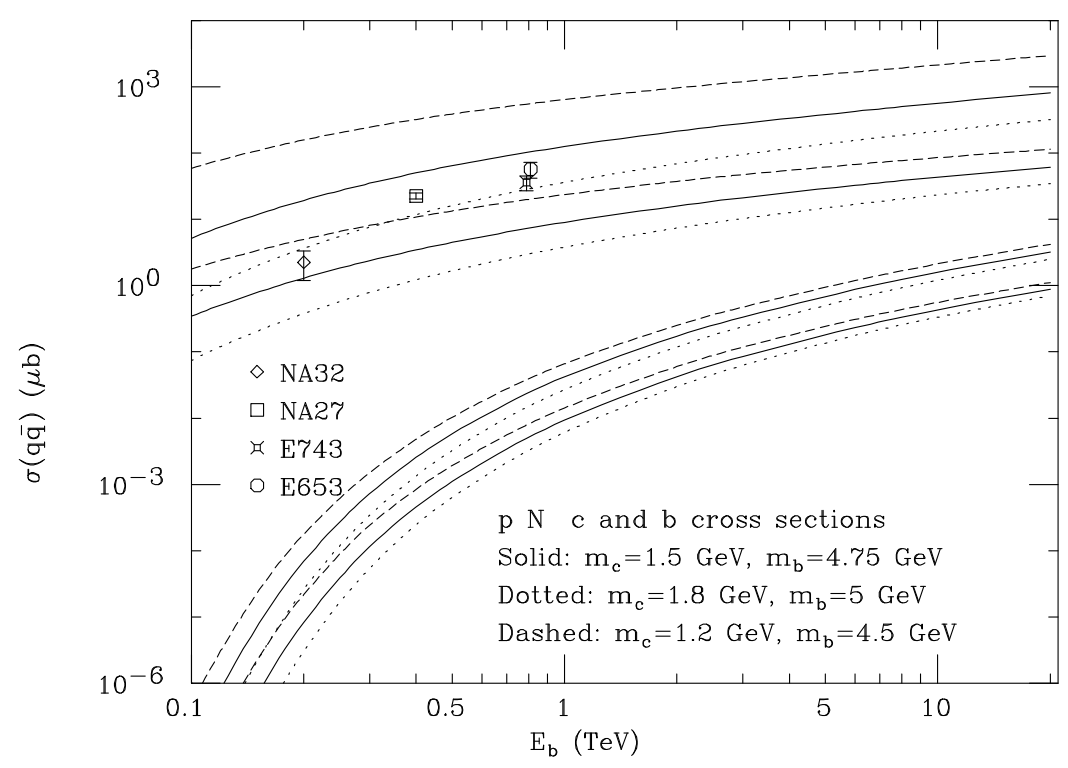

Figure 2: Cross sections for $b$ and $c$ production in $p N$ collisions versus experimental results.

The choice of a range of values for $\Lambda_{4}$ requires some special considerations. The values of $\Lambda_{4}$ obtained in the fits of ref. [27] range from 135 to $235 \mathrm{MeV}$, corresponding to a range for $\Lambda_{5}$ between 84 and $155 \mathrm{MeV}$. This range for $\Lambda_{4}$ is chosen because no 
good fit to deep inelastic data is possible outside that range in the context of ref. [27] (i.e. with that choice of structure function parametrization, etc.). However, LEP data favour larger values of $\Lambda$, the typical value being $\Lambda_{5} \simeq 300 \mathrm{MeV}$, which corresponds to $\Lambda_{4} \simeq 400 \mathrm{MeV}$ [28]. For this reason, we have stretched the $\Lambda_{4}$ range proposed in ref. [27] to $100<\Lambda_{4}<300 \mathrm{MeV}$, corresponding to $60<\Lambda_{5}<204 \mathrm{MeV}$. Since the pion structure functions we are using [26] are fitted with a fixed value of $\Lambda_{4}=190$ $\mathrm{MeV}$, corresponding to $\Lambda_{5}=122 \mathrm{MeV}$, we prefer not to stretch the $\Lambda_{5}$ range up to the LEP value of $300 \mathrm{MeV}$. Therefore, in order to take into account the full range of uncertainty associated to the value of $\Lambda$, we were forced to account only partially for the correlation between $\Lambda$ and the nucleon and pion structure functions. On the other hand, in ref. [29] a fit of the parton densities with a fixed value of $\Lambda_{5}=220$ $\mathrm{MeV}$ was performed. We checked that with this set our results are very close to those obtained with the MRS235 set and $\Lambda_{4}=300 \mathrm{MeV}$.

Observe the considerable improvement in predictivity, after inclusion of next-toleading-order corrections, that takes place when going from charm to bottom. Observe also the strong mass dependence of the charm result.

The results of the same analysis for a proton beam are shown in fig. 2. Most of the considerations made in the case of the pion beam also apply to the case of protons. This is certainly the case for the large overall range of values allowed by the uncertainties of the calculation.

Experimental results on bottom production at fixed target have been reported in refs. [30-35]. These results are also shown in fig. 1 . We made no effort to correct the data in order to get the $b \bar{b}$ quark cross section. The E653 collaboration quotes directly a $b \bar{b}$ cross section.

As one can see, experimental results on total cross sections for charm and bottom production at fixed target are in reasonable agreement with theoretical expectations, if the large theoretical uncertainties are taken into proper account. We can see that the hadroproduction data are compatible with a value of $1.5 \mathrm{GeV}$ for the charm quark mass. We remind the reader that many puzzling ISR results in $p p$ collisions at $62 \mathrm{GeV}$ remain difficult to explain (see the review in ref. [36]), in particular the large $\Lambda_{b}$ production rates reported in ref. [30].

Total cross sections for charm production have also been measured in photoproduction experiments. In fig. 3 the relevant experimental results of refs. $[21,37,38]$ 


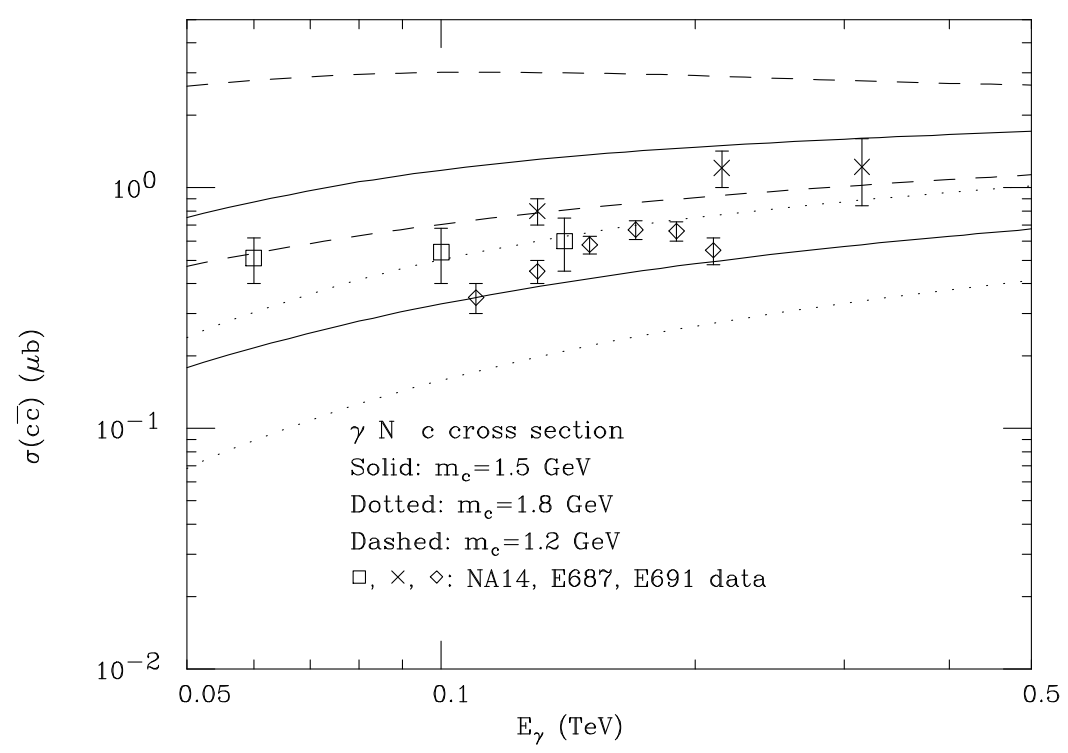

Figure 3: Cross sections for c production in $\gamma N$ collisions versus experimental results.

are shown in comparison to next-to-leading QCD predictions. As can be seen, the theoretical uncertainties are smaller in this case than in the hadroproduction case. Again, a charm mass of $1.5 \mathrm{GeV}$ is compatible with photoproduction data.

\section{Single-inclusive differential distributions}

\subsection{Charm hadroproduction}

We have collected in table 2 some of the most recent experimental measurements of $p_{T}^{2}$ and $x_{F}$ single-inclusive distributions in charm hadroproduction. The experimental collaborations fit their data using the following parametrization:

$$
\frac{d \sigma}{d x_{F}}=A\left(1-x_{F}\right)^{n}
$$

for the $x_{F}$ distribution, and

$$
\frac{d \sigma}{d p_{T}^{2}}=C e^{-b p_{T}^{2}}
$$




\begin{tabular}{|l||c|c|c|c|}
\hline Collaboration & $\begin{array}{c}\text { Beam } \\
\text { Target }\end{array}$ & $\begin{array}{c}E_{b} \\
(\mathrm{GeV})\end{array}$ & $n(D / \bar{D})$ & $\begin{array}{c}b(D / \bar{D}) \\
\left(\mathrm{GeV}^{-2}\right)\end{array}$ \\
\hline \hline E653 [13] & $p N(\mathrm{E})$ & 800 & $6.9_{-1.8}^{+1.9}$ & $0.84_{-0.08}^{+0.10}$ \\
\hline NA32 (ACCMOR) [14] & $p N(\mathrm{Si})$ & 200 & $5.5_{-1.8}^{+2.1}$ & $1.4_{-0.4}^{+0.6}$ \\
\hline E743 (LEBC-MPS) [15] & $p p\left(\mathrm{H}_{2}\right)$ & 800 & $8.6 \pm 2.0$ & $0.8 \pm 0.2$ \\
\hline NA27 (LEBC-EHS) [16] & $p p\left(\mathrm{H}_{2}\right)$ & 400 & $4.9 \pm 0.5$ & $0.99 \pm 0.09$ \\
\hline WA82 [39] & $p N(\mathrm{Cu})$ & 370 & $6.0 \pm 0.3$ & $0.93 \pm 0.09$ \\
\hline E653 [19] & $\pi^{-} N(\mathrm{E})$ & 600 & $4.25 \pm 0.33$ & $0.76 \pm 0.04$ \\
\hline NA32 (ACCMOR) [18] & $\pi^{-} N(\mathrm{Cu})$ & 230 & $3.74 \pm 0.23$ & $0.83 \pm 0.03$ \\
\hline NA32 (ACCMOR) [14] & $\pi^{-} N(\mathrm{Si})$ & 200 & $2.5_{-0.3}^{+0.4}$ & $1.06_{-0.11}^{+0.12}$ \\
\hline NA27 (LEBC-EHS) [17] & $\pi^{-} p(\mathrm{H} 2)$ & 360 & $3.80 \pm 0.63$ & $1.18_{-0.16}^{+0.18}$ \\
\hline E769 [40] & $\pi^{-} N(\mathrm{Be})$ & 250 & $3.9 \pm 0.3$ & $1.03 \pm 0.06$ \\
\hline WA82 [39] & $\pi^{-} N(\mathrm{Si})$ & 340 & $2.9 \pm 0.3$ & $0.86 \pm 0.05$ \\
\hline WA75 [41] & $\pi^{-} N(\mathrm{E})$ & 350 & $3.5 \pm 0.5$ & $0.77 \pm 0.04$ \\
\hline
\end{tabular}

Table 2: Experimental results for single-inclusive distributions for D-meson production. WA82 used also $W$ targets in $p N$ collisions, and $C u$ and $W$ in $\pi^{-} N$ collisions. E769 used also $\mathrm{Al}, \mathrm{Cu}, \mathrm{W}$ to study atomic-number dependence.

for the $p_{T}^{2}$ distribution. The data are then presented in the form of measured values for the parameters $n$ and $b$, which are reported in the last two columns of table 2 . A possible way of comparing the experimental results with QCD predictions is that of fitting the theoretical distributions using the same functional forms, eqs. (3.1) and (3.2), and then comparing the values of the fit parameters obtained in this way with the measured ones. We have therefore fitted the $x_{F}$ and $p_{T}^{2}$ single-inclusive distributions for charm production computed at next-to-leading order in perturbative QCD, using the forms in eqs. (3.1) and (3.2). We have computed the distributions with an $x_{F}>0$ cut, in order to compare with experimental data. The results of these fits are presented in tables 3 and 4 for pion-nucleon and proton-nucleon collisions respectively. The dependence of the fit parameters upon renormalization scale, parton densities, charm mass and beam energy is also shown. The default values (marked 


\begin{tabular}{|l||c||c|c||c|c||c|c||c|c|}
\hline \multicolumn{1}{|l||}{} & \multicolumn{1}{|c||}{} & \multicolumn{2}{c||}{$\mu_{R}$} & \multicolumn{2}{c||}{$\Lambda_{5}(\mathrm{GeV})$} & \multicolumn{2}{c||}{$m_{c}(\mathrm{GeV})$} & \multicolumn{2}{c|}{$E_{b}(\mathrm{GeV})$} \\
\hline & DEF & $2 \mu_{0}$ & $\mu_{0} / 2$ & 0.06 & 0.204 & 1.2 & 1.8 & 230 & 600 \\
\hline \hline$n$ & 4.24 & 4.30 & 4.13 & 4.29 & 4.20 & 4.60 & 3.98 & 3.85 & 4.91 \\
\hline \hline$b\left(p_{T}^{2}<10 \mathrm{GeV}^{2}\right)$ & 0.623 & 0.609 & 0.648 & 0.598 & 0.647 & 0.730 & 0.530 & 0.679 & 0.563 \\
\hline$b\left(p_{T}^{2}<5 \mathrm{GeV}^{2}\right)$ & 0.801 & 0.779 & 0.827 & 0.768 & 0.827 & 0.986 & 0.666 & 0.852 & 0.729 \\
\hline$b\left(p_{T}^{2}<3 \mathrm{GeV}^{2}\right)$ & 0.928 & 0.909 & 0.977 & 0.893 & 0.970 & 1.171 & 0.756 & 1.001 & 0.864 \\
\hline
\end{tabular}

Table 3: Values of the parameters $n$ and $b$ in the fits of the $x_{F}$ and $p_{T}^{2}$ distributions, for various choices of $\mu_{R}$, beam energy, mass, and structure functions, (as discussed in the text) in pion-nucleon collisions.

\begin{tabular}{|l||c||c|c||c|c||c|c||c|}
\hline \multicolumn{1}{|l||}{} & \multicolumn{1}{|c||}{} & \multicolumn{2}{c||}{$\mu_{R}$} & \multicolumn{2}{c||}{$\Lambda_{5}(\mathrm{GeV})$} & \multicolumn{2}{c||}{$m_{c}(\mathrm{GeV})$} & $E_{b}(\mathrm{GeV})$ \\
\hline & DEF & $2 \mu_{0}$ & $\mu_{0} / 2$ & 0.06 & 0.204 & 1.2 & 1.8 & 400 \\
\hline \hline$n$ & 8.36 & 8.50 & 8.14 & 7.97 & 8.45 & 8.31 & 7.88 & 7.35 \\
\hline \hline$b\left(p_{T}^{2}<10 \mathrm{GeV}^{2}\right)$ & 0.635 & 0.627 & 0.649 & 0.599 & 0.670 & 0.730 & 0.533 & 0.754 \\
\hline$b\left(p_{T}^{2}<5 \mathrm{GeV}^{2}\right)$ & 0.799 & 0.783 & 0.824 & 0.759 & 0.833 & 0.977 & 0.668 & 0.904 \\
\hline$b\left(p_{T}^{2}<3 \mathrm{GeV}^{2}\right)$ & 0.930 & 0.903 & 0.976 & 0.882 & 0.982 & 1.155 & 0.747 & 1.059 \\
\hline
\end{tabular}

Table 4: Values of the parameters $n$ and $b$ in the fits of the $x_{F}$ and $p_{T}^{2}$ distributions, for various choices of $\mu_{R}$, beam energy, mass, and structure functions (as discussed in the text) in proton-nucleon collisions.

DEF in the tables) are $m_{c}=1.5 \mathrm{GeV}, \Lambda_{5}=0.122 \mathrm{GeV}$ (used in conjunction with the structure function set HMRSB for the nucleon and SMRS2 for the pion), $E_{b}=$ $350 \mathrm{GeV}$ for incident pions, $E_{b}=800 \mathrm{GeV}$ for incident protons, $\mu_{F}=2 \mu_{0}, \mu_{R}=\mu_{0}$, $\mu_{0}=\sqrt{p_{T}^{2}+m_{c}^{2}}$. The parameters were varied one at the time, as indicated in the tables. The low (high) value of $\Lambda_{5}$ was used in conjunction with the structure function set MRS135 (MRS235).

We observe that the parameter $b$, which characterizes the $p_{T}^{2}$ distributions, is very sensitive to the upper bound of the $p_{T}^{2}$ range. This is due to the fact that the fall-off 
of the cross section at large $p_{T}$ is not exponential, but rather follows a power law. Experimental data show a similar trend (see for example fig. 8 in ref. [39]). We find that the form

$$
\frac{d \sigma}{d p_{T}^{2}}=\left(\frac{C}{b m_{c}^{2}+p_{T}^{2}}\right)^{\beta}
$$

provides an excellent fit to the theoretical distributions in the whole $p_{T}$ range. This is illustrated in fig. 4, where a $p_{T}^{2}$ distribution computed in next-to-leading-order QCD (for $\pi N$ collisions at $E_{b}=250 \mathrm{GeV}$ ) is shown, together with the corresponding fits obtained with eqs. (3.2) and (3.3). The results of the fits in the form of eq. (3.3) are

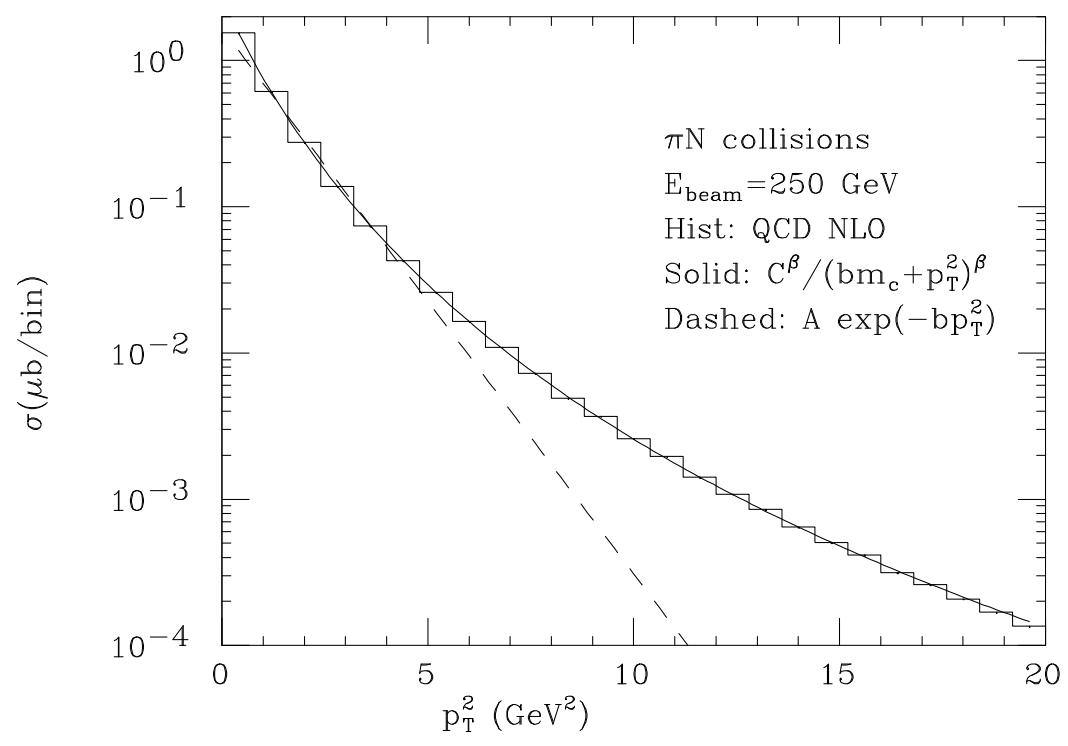

Figure 4: The calculated $p_{T}$ single-inclusive heavy-quark distribution, and the corresponding fits illustrated in the text.

given in tables 5 for $\pi N$ and 6 for $p N$ collisions. Both tables are obtained with an $x_{F}>0$ cut.

We have also fitted the theoretical single-inclusive $x_{F}$ and $p_{T}^{2}$ distributions (for $p_{T}^{2}$ up to $3 \mathrm{GeV}^{2}$ ) in the form of eqs. (3.1) and (3.2) for a wide range in the beam energy, both for pion-nucleon and proton-nucleon collisions. The results are reported, as plots of $n$ and $b$ versus beam energy, in figs. 5 to 9 , together with the experimental values of $n$ and $b$ reported in table 2. The dependence of $n$ and $b$ upon the charm quark mass is also displayed. All other parameters are kept fixed at the default 


\begin{tabular}{|c||c||c|c||c|c||c|c||c|c|}
\hline \multicolumn{1}{|l||}{} & \multicolumn{1}{c||}{} & \multicolumn{2}{c||}{$\mu_{R}$} & \multicolumn{2}{c||}{$\Lambda_{5}(\mathrm{GeV})$} & \multicolumn{2}{c|}{$m_{c}(\mathrm{GeV})$} & \multicolumn{2}{c|}{$E_{b}(\mathrm{GeV})$} \\
\hline & DEF & $2 \mu_{0}$ & $\mu_{0} / 2$ & 0.06 & 0.204 & 1.2 & 1.8 & 230 & 600 \\
\hline \hline$C$ & 5.281 & 4.856 & 5.831 & 4.905 & 5.572 & 4.868 & 5.744 & 4.883 & 5.855 \\
\hline$b$ & 1.795 & 1.799 & 1.768 & 1.871 & 1.704 & 1.933 & 1.719 & 1.795 & 1.802 \\
\hline$\beta$ & 5.220 & 5.109 & 5.392 & 5.143 & 5.247 & 5.024 & 5.413 & 5.600 & 4.807 \\
\hline
\end{tabular}

Table 5: Values of the parameters $C, b$ and $\beta$ in the fits of the $p_{T}^{2}$ distributions of the form $C^{\beta} /\left(b m_{c}^{2}+p_{T}^{2}\right)^{\beta}$, for various choices of $\mu_{R}$, beam energy, mass, and structure functions (as discussed in the text) in pion-nucleon collisions. Cross sections are expressed in $\mu b$, masses and momenta in GeV.

\begin{tabular}{|c||c||c|c||c|c||c|c||c|}
\hline \multicolumn{1}{|l||}{} & \multicolumn{1}{|c||}{} & \multicolumn{2}{c||}{$\mu_{R}$} & \multicolumn{2}{c||}{$\Lambda_{5}(\mathrm{GeV})$} & \multicolumn{2}{c|}{$m_{c}(\mathrm{GeV})$} & $E_{b}(\mathrm{GeV})$ \\
\hline & DEF & $2 \mu_{0}$ & $\mu_{0} / 2$ & 0.06 & 0.204 & 1.2 & 1.8 & 400 \\
\hline \hline$C$ & 6.503 & 6.106 & 7.004 & 6.089 & 6.750 & 5.849 & 7.298 & 6.174 \\
\hline$b$ & 1.966 & 2.043 & 1.903 & 2.070 & 1.890 & 2.089 & 1.963 & 2.228 \\
\hline$\beta$ & 5.540 & 5.513 & 5.580 & 5.377 & 5.603 & 5.209 & 5.896 & 6.788 \\
\hline
\end{tabular}

Table 6: Values of the parameters $C, b$ and $\beta$ in the fits of the $p_{T}^{2}$ distributions of the form $C^{\beta} /\left(b m_{c}^{2}+p_{T}^{2}\right)^{\beta}$, for various choices of $\mu_{R}$, beam energy, mass, and structure functions (as discussed in the text) in proton-nucleon collisions. Cross sections are expressed in $\mu b$, masses and momenta in GeV.

values. We have verified that changing the other input parameters (parton densities, renormalization scale) has an effect on the computed values of $n$ and $b$, which is in general negligible when compared to the heavy-quark mass dependence. For this reason, we did not include these effects in the figures. As can be seen from figs. 5 and 7 , the experimental measurements seem to suggest values of $n$ that are smaller than the purely perturbative QCD result, both for the pion and the proton cases. In principle, some description of the hadronization phenomena should be added to the perturbative calculation in order to compare it with the data. These problems were considered in ref. [12], where the hadronization phenomena were studied using the 


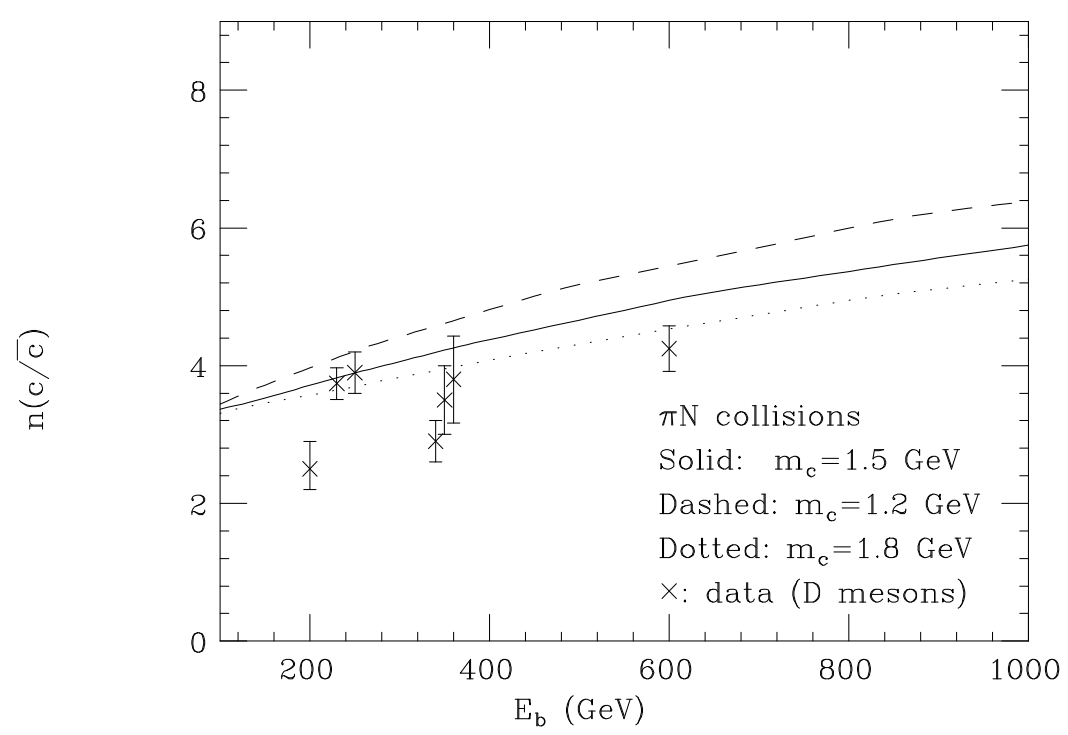

Figure 5: Theoretical predictions for the parameter $n$ in pion-nucleon charm production for different $m_{c}$ values. Experimental results are also reported.

parton shower Monte Carlo HERWIG. In ref. [12] the conclusion was reached that the combined effects of perturbative higher orders and nonperturbative (partonic intrinsic transverse momentum and hadronization) contributions eventually result in a hardening of the $x_{F}$ distribution, that is, in a smaller value of $n$. In ref. [12] it was also argued that the usual approach of complementing the perturbative calculation with a fragmentation function in order to describe the $x_{F}$ distribution is completely unjustified, since the factorization theorem holds only in the large- $p_{T}$ region.

Almost all the experimental collaborations observe, in pion-nucleon collisions, the so-called leading particle effect, that is, an enhanced production of the $D$-mesons whose light valence quark is of the same flavour as one of the valence quarks of the incoming pion. From fig. 6 we see that the QCD prediction is in better agreement with the available data for non-leading particles (with the exception of the NA27 measurement, which has, however, a large uncertainty) than in the case of the full $D$-meson sample. For proton-nucleon collisions the situation is less clear. Some collaborations explicitly state that no leading effect is observed. Another possible explanation of the hardness of the $x_{F}$ distribution was put forward in ref. [42], where it was shown that higher-twist effects, suppressed by inverse powers of the heavy- 


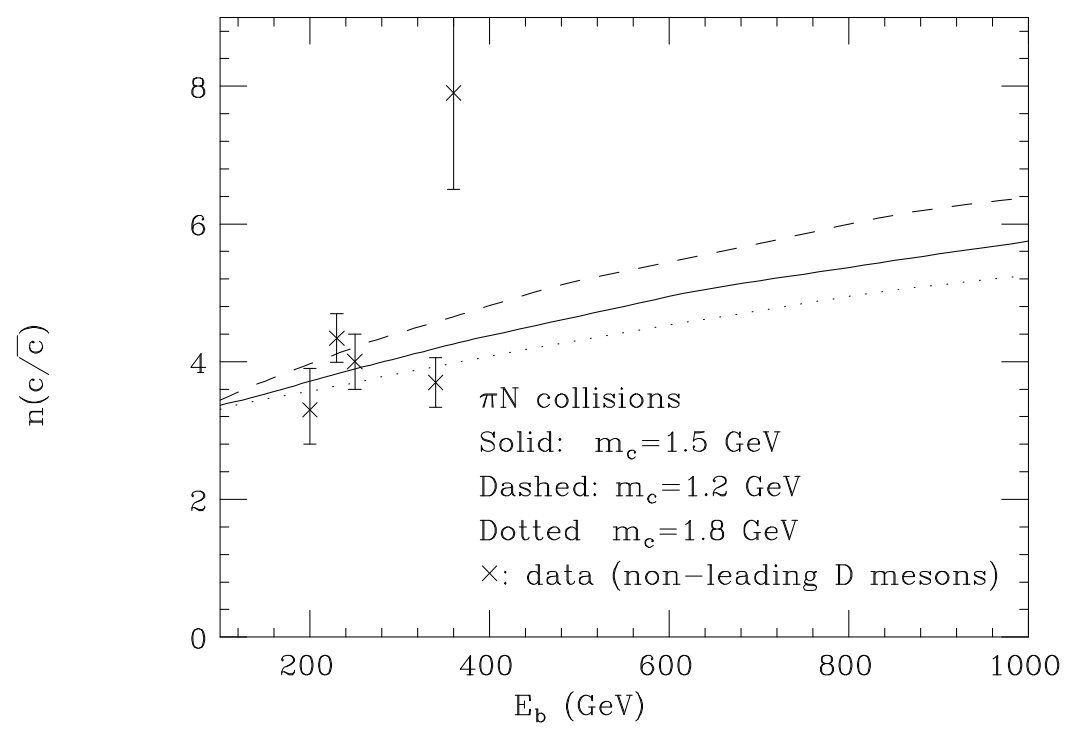

Figure 6: Theoretical predictions for the parameter $n$ in pion-nucleon charm production for different $m_{c}$ values. The experimental results are for the non-leading D-meson production.

quark mass, but enhanced by inverse powers of $1-x_{F}$, are present, and that they could provide an enhancement of the cross section in the large- $x_{F}$ region.

The use of the parameters $n$ and $b$ for comparing the data with the theory is not entirely satisfactory. In fact, the fits to experimental data are influenced by statistical uncertainties, which obviously differ from one bin to another in the same histogram. On the other hand, fits to theoretical distributions are performed with a uniform, arbitrarily small error. The two procedures may lead to different results, due to the fact that the functional forms in eqs. (3.1) and (3.2) do not fit the theoretical predictions well, and in some cases not even the data. It seems therefore more significant to compare the experimental and theoretical distributions directly. This is done in figs. 10 and 11 for the single-inclusive $p_{T}^{2}$ distribution measured by the WA82 [39] and E769 [40] in $\pi N$ collisionsat $E_{b}=350 \mathrm{GeV}$ and $250 \mathrm{GeV}$. respectively. In both cases, the agreement with the next-to-leading-order QCD calculation is almost perfect over the whole $p_{T}^{2}$ range explored by the experiment, although no higher-order or nonperturbative effects are included in this theoretical curve. This implies that the data are well fitted by the form in eq. (3.3), since this form gives a good fit to the theoretical 


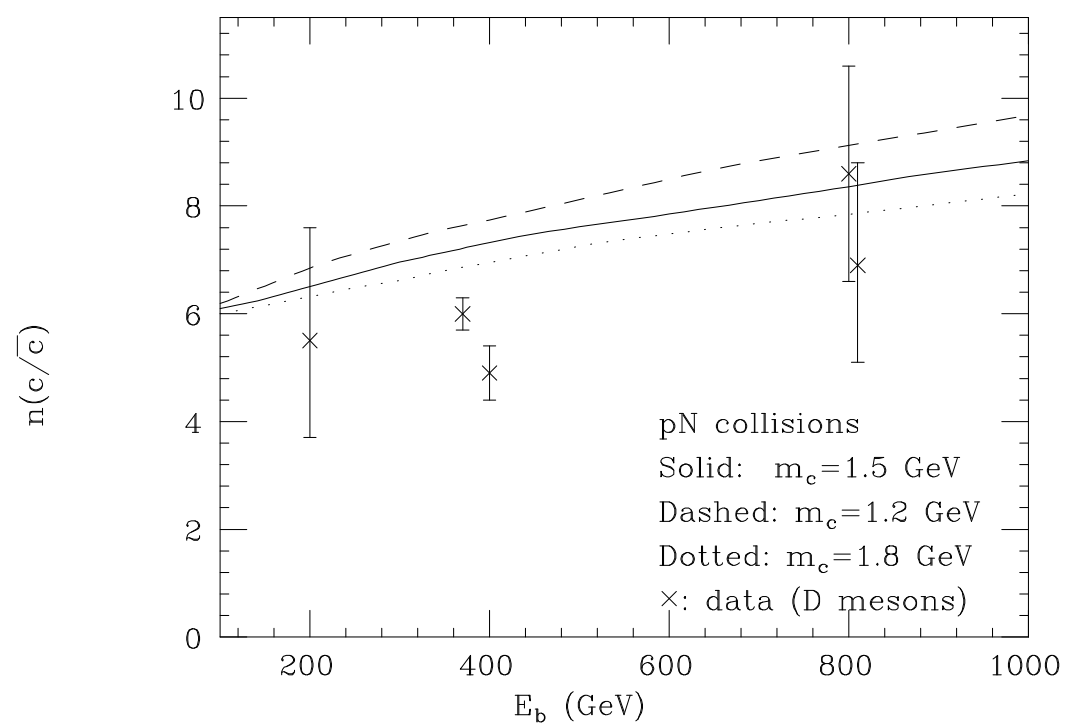

Figure 7: Theoretical predictions for the parameter $n$ in proton-nucleon charm production for different $m_{c}$ values versus experimental results.

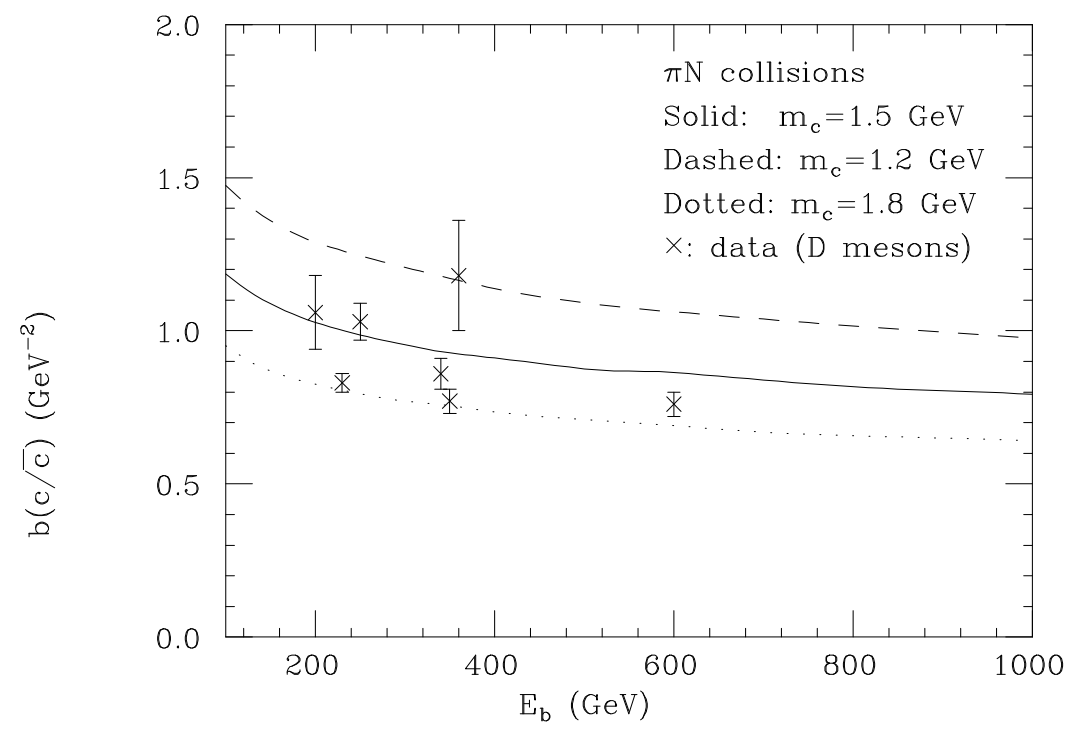

Figure 8: Theoretical predictions for the parameter $b$ in pion-nucleon charm production for different $m_{c}$ values versus experimental results.

distributions. 


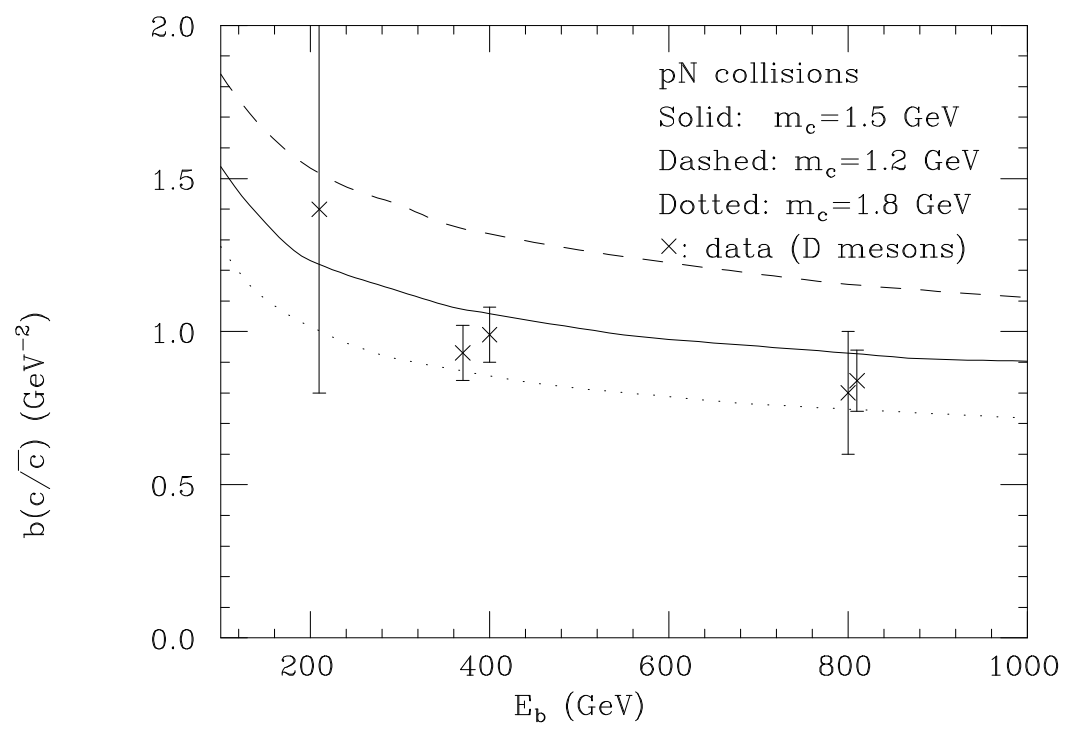

Figure 9: Theoretical prediction for the parameter $b$ versus experimental results in proton-nucleon collisions.

Due to the low mass of the charm quark, we can however expect that nonperturbative effects, like an intrinsic transverse momentum of the incoming partons, may play an important rôle. We therefore included in our calculation an intrinsic transverse momentum for the incoming partons, in the following way. For each event, in the longitudinal CM frame of the heavy-quark pair, we perform a Lorentz boost in the transverse plane, to the frame where the transverse momentum of the heavy-quark pair, $\vec{p}_{T}(Q \bar{Q})$, is equal to zero. We then perform a second transverse boost that gives the pair a transverse momentum equal to $\vec{p}_{T}(Q \bar{Q})+\vec{k}_{T}(1)+\vec{k}_{T}(2)$, where $\vec{k}_{T}(1)$ and $\vec{k}_{T}(2)$ are the transverse momenta of the incoming partons, whose modulus is randomly distributed according to the Gaussian law

$$
\frac{1}{N} \frac{d N}{d k_{T}^{2}}=\frac{1}{\left\langle k_{T}^{2}\right\rangle} \exp \left(-k_{T}^{2} /\left\langle k_{T}^{2}\right\rangle\right) .
$$

The inclusion of this effect results in a hardening of the single-inclusive $p_{T}^{2}$ distribution.

Another nonperturbative effect that must be accounted for is the hadronization process. Thanks to the factorization theorem, this effect can be described by convoluting the partonic cross section with a fragmentation function, which we choose to be of the Peterson form [43]. This degrades the parent charm-quark momentum, and 


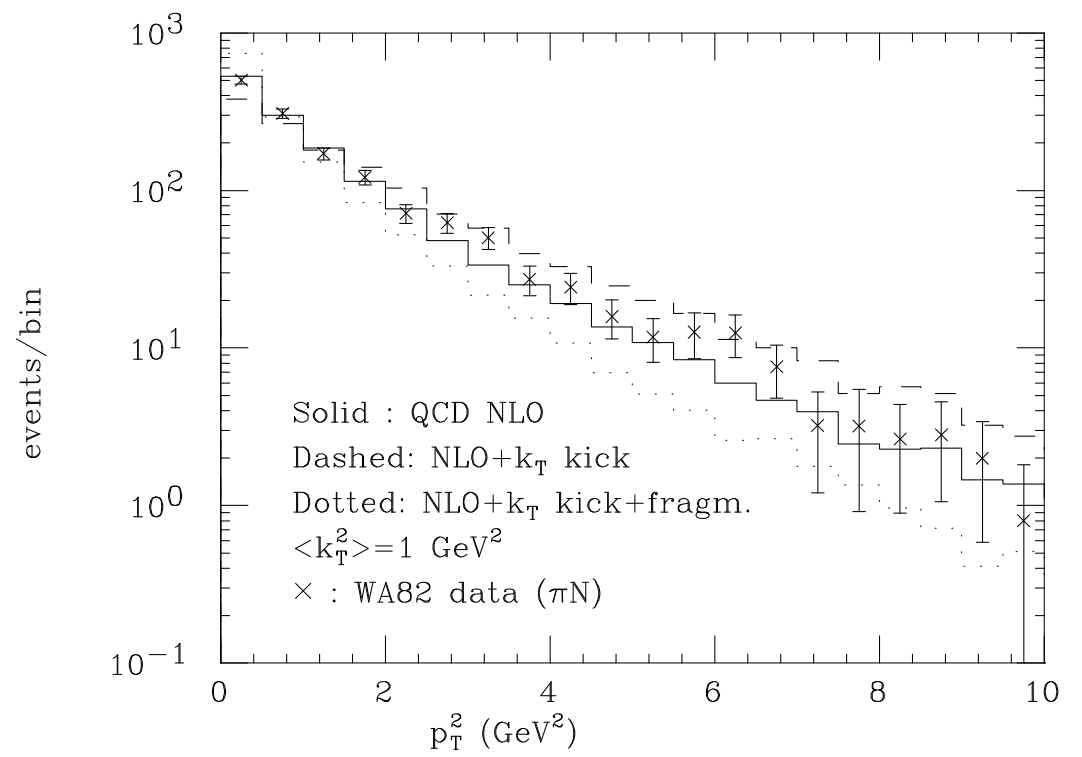

Figure 10: The single-inclusive $p_{T}^{2}$ distribution measured by WA82, compared to the next-to-leading order QCD prediction, with and without the inclusion of nonperturbative effects.

results in a softening of the $p_{T}$ distribution.

Both effects are shown in figs. 10-11, for $\left\langle k_{T}^{2}\right\rangle=1 \mathrm{GeV}^{2}$. Since our next-to-leading calculation already includes part of the effect of the evolution of the fragmentation function, our Peterson form should refer to hadron formation from a quark which has already evolved to small virtuality. In this case, an appropriate choice for the parameter $\epsilon_{c}$ that characterizes the Peterson fragmentation function is $\epsilon_{c}=0.06$. We have verified that the result does not change substantially if we use the smaller value $\epsilon_{c}=0.04$. From inspection of figs. 10-11, we can conclude that perturbative QCD, supplemented with some parametrization of the most important nonperturbative effects, leads to a prediction in qualitative agreement with the experimental singleinclusive $p_{T}^{2}$ distribution measured by the WA82 and E769 collaborations. However, we have checked that, in order to reproduce the WA82 and E769 data, an average intrinsic transverse momentum $\left\langle k_{T}^{2}\right\rangle=2 \mathrm{GeV}^{2}$ is needed. This value for $\left\langle k_{T}^{2}\right\rangle$ is rather large, and we will comment in due time upon its effect on other observables. One may attempt to use larger values of the charm quark mass in order to get better agreement with data without the need for a large $\left\langle k_{T}^{2}\right\rangle$. In fact, a larger $m_{c}$ would harden the 


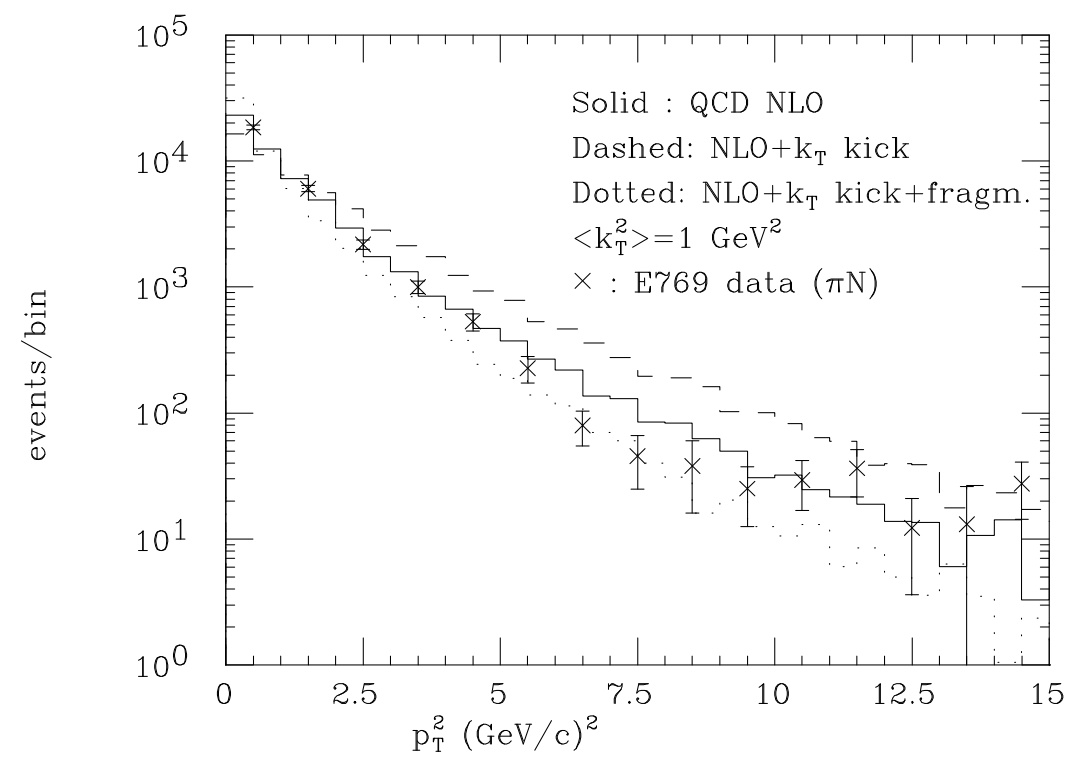

Figure 11: The single-inclusive $p_{T}^{2}$ distribution measured by E769, compared to the next-to-leading order QCD prediction, with and without the inclusion of nonperturbative effects.

$p_{T}$ spectrum of the quark. As better data will become available, this will certainly be worth doing.

In figs. 12 and 13 we present the $x_{F}$ distributions measured by WA82 in $\pi N$ collisions, for $D^{-}$and $D^{+}$mesons respectively. Both distributions are compared with the same theoretical curve for charm quark, obtained with the purely perturbative next-to-leading order QCD calculation. We can see that the experimental data show a harder behaviour, and that the agreement with the theoretical distribution is satisfactory in the case of the non-leading hadron ( $D^{+}$in this case), as already observed in commenting fig. 6. This is another indication that nonperturbative phenomena (such as colour drag effects) are present in the production of leading particles.

\subsection{Charm photoproduction}

Single-inclusive distributions for charm production have also been measured by photonnucleon collision experiments. In the case of photoproduction, we expect QCD pre- 


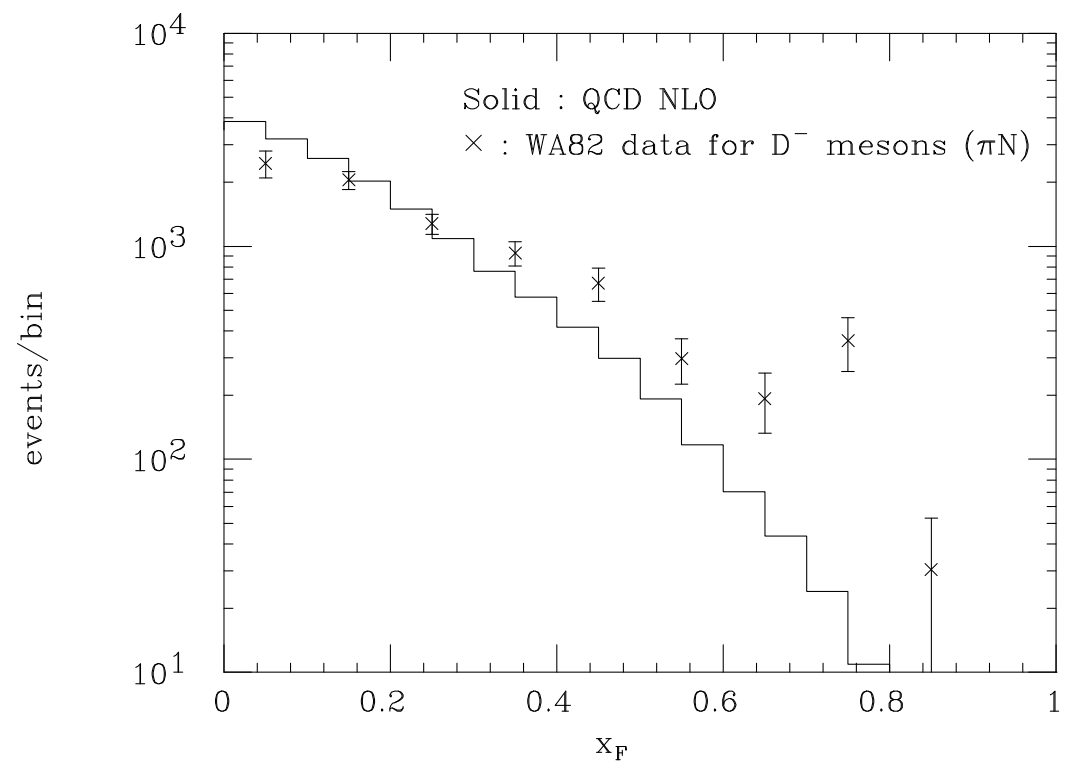

Figure 12: Experimental $x_{F}$ distribution for $D^{-}$mesons, compared to the next-to-leading-order QCD prediction for charm quarks.

dictions to be more reliable than in the hadroproduction case, since only one hadron is present in the initial state (see refs. [7] and [10] for a detailed discussion).

The E691 [37] and E687 [38] collaborations have measured the single-inclusive $p_{T}^{2}$ distribution, and report their results in the form of measured values for the parameters $a_{1}$ and $a_{2}$ that appear in the expression they use to fit the data:

$$
\frac{d \sigma}{d p_{T}^{2}}=A e^{-a_{1} p_{T}^{2}+a_{2} p_{T}^{4}}
$$

Their results are

$$
\begin{aligned}
& \text { E687 : } \quad a_{1}=0.85 \pm 0.02 \mathrm{GeV}^{-2} ; \quad a_{2}=0.03 \pm 0.01 \mathrm{GeV}^{-4} \\
& \text { E691 : } a_{1}=1.07 \pm 0.05 \mathrm{GeV}^{-2} ; \quad a_{2}=0.04 \pm 0.01 \mathrm{GeV}^{-4} \text {. }
\end{aligned}
$$

We have fitted, with the same parametrization of eq. (3.5), the next-to-leading QCD distribution, supplemented with Peterson fragmentation, and with an intrinsic $k_{T}$ for the incoming partons as in the case of hadroproduction. Equation (3.5) can only be used in a limited range for $p_{T}^{2}$. We chose to perform the fits for $p_{T}^{2}<10$ 


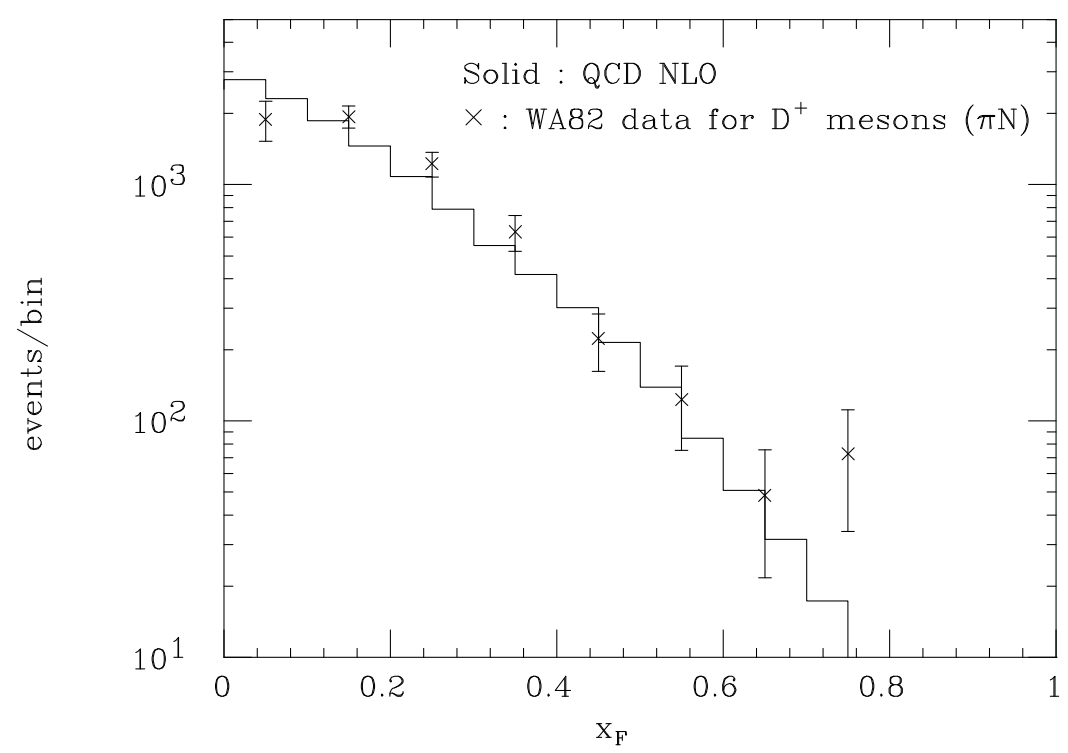

Figure 13: Experimental $x_{F}$ distribution for $D^{+}$mesons, compared to the next-to-leading-order QCD prediction for charm quarks.

\begin{tabular}{|c||c|c|c||c|c|c|}
\hline \multicolumn{1}{|c||}{} & \multicolumn{3}{c||}{ E687 } & \multicolumn{3}{c|}{ E691 } \\
\hline & $\begin{array}{c}\text { QCD } \\
\text { NLO }\end{array}$ & $\begin{array}{c}\left\langle k_{T}^{2}\right\rangle=0.5 \mathrm{GeV}^{2} \\
+ \text { fragm. }\end{array}$ & $\begin{array}{c}\left\langle k_{T}^{2}\right\rangle=1 \mathrm{GeV}^{2} \\
+ \text { fragm. }\end{array}$ & $\begin{array}{c}\text { QCD } \\
\text { NLO }\end{array}$ & $\begin{array}{c}\left\langle k_{T}^{2}\right\rangle=0.5 \mathrm{GeV}^{2} \\
+ \text { fragm. }\end{array}$ & $\begin{array}{c}\left\langle k_{T}^{2}\right\rangle=1 \mathrm{GeV}^{2} \\
+ \text { fragm. }\end{array}$ \\
\hline \hline$a_{1}$ & 0.656 & 1.012 & 0.947 & 0.721 & 1.071 & 1.025 \\
\hline$a_{2}$ & 0.021 & 0.039 & 0.035 & 0.022 & 0.038 & 0.036 \\
\hline \hline$C$ & 5.703 & 2.278 & 2.539 & 6.445 & 2.574 & 2.688 \\
\hline$b$ & 3.072 & 1.041 & 1.196 & 3.396 & 1.205 & 1.286 \\
\hline$\beta$ & 5.010 & 4.046 & 4.185 & 6.075 & 4.823 & 4.759 \\
\hline
\end{tabular}

Table 7: Values of the parameters $a_{1}, a_{2}$ and $C, b$ and $\beta$ for the fits to the $p_{T}^{2}$ distribution, at E687 and E691 energies.

$\mathrm{GeV}^{2}$. Our results are presented in table 7 . The input parameters are the default parameters we used in the hadroproduction case. In both cases, we have used the shape of the photon beam spectrum of E687, with an average energy $\left\langle E_{\gamma}\right\rangle=220$ 


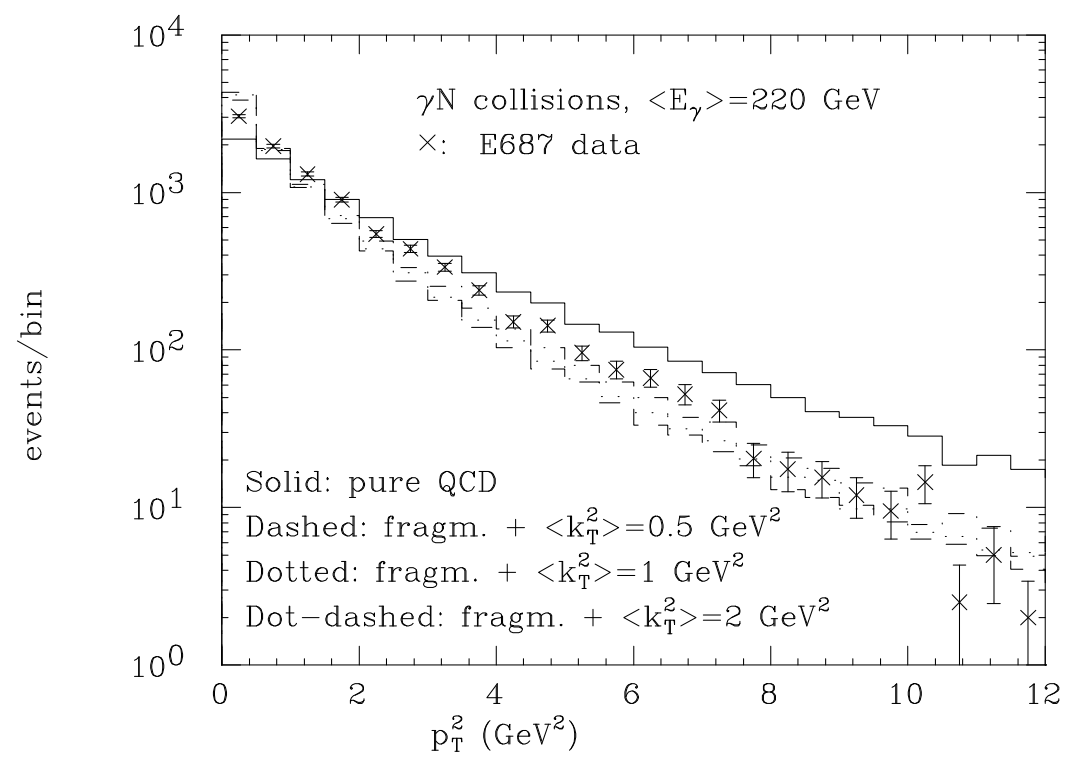

Figure 14: Experimental $p_{T}^{2}$ distribution compared to the next-to-leading order QCD prediction, with and without the inclusion of nonperturbative effects, in $\gamma N$ collisions at $\left\langle E_{\gamma}\right\rangle=220 \mathrm{GeV}$.

$\mathrm{GeV}$ for the E687 experiment and $\left\langle E_{\gamma}\right\rangle=145 \mathrm{GeV}$ for E691. In performing these fits, the error on the theoretical distribution has been kept constant over the whole $p_{T}^{2}$ range considered. The agreement with the experimental values, eqs. (3.6) and (3.7), is remarkably good for E691, and reasonably good for E687. We have also fitted the theoretical $p_{T}^{2}$ distributions using the form in eq. (3.3). The corresponding parameters $C, b$ and $\beta$ are shown in table 7. The form in eq. (3.3) has the advantage that it can be used over an arbitrarily large $p_{T}^{2}$ range.

In figs. 14 and 15 we show the $p_{T}^{2}$ distributions measured by the E687 and E691 collaborations respectively. We also show the next-to-leading-order QCD prediction, and the QCD prediction supplemented with Peterson fragmentation and an intrinsic transverse momentum for the incoming partons with $\left\langle k_{T}^{2}\right\rangle=0.5 \mathrm{GeV}^{2}, 1 \mathrm{GeV}^{2}$ and $2 \mathrm{GeV}^{2}$. It is interesting to notice that, in this case, the fragmentation effect, combined with a moderate intrinsic transverse momentum of the initial-state partons, is sufficient to reproduce the experimental distribution. Contrary to what happens in the hadroproduction case, this distribution is now less sensitive to the choice of the 


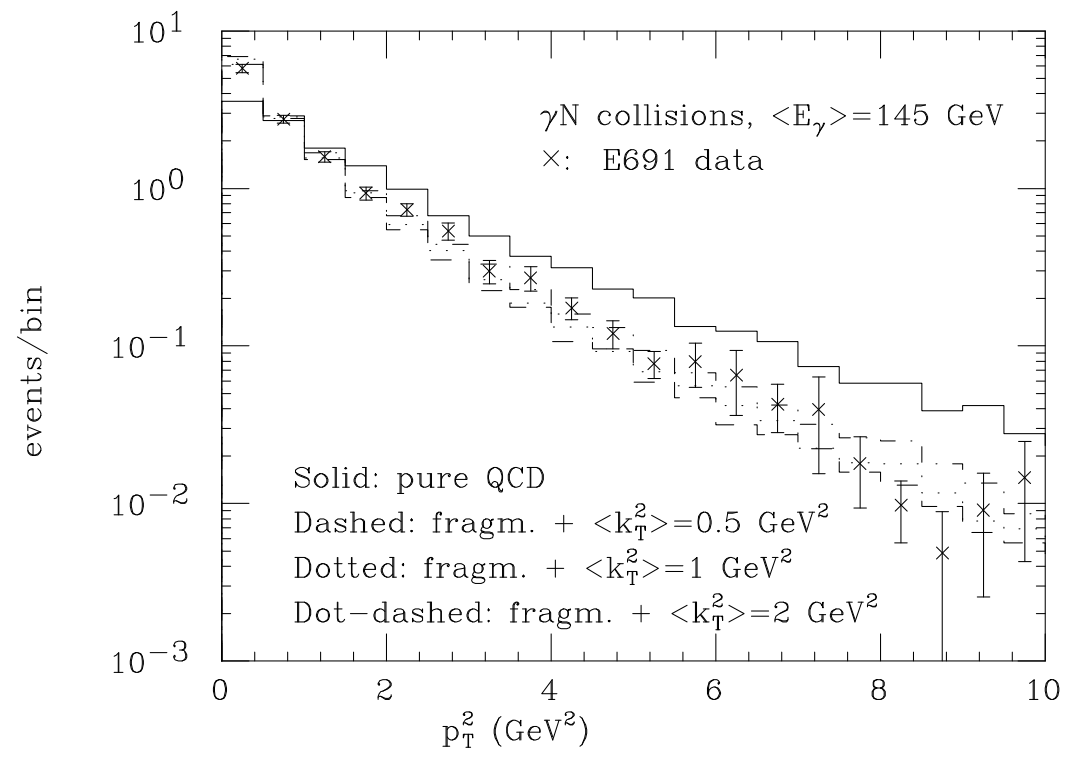

Figure 15: Experimental $p_{T}^{2}$ distribution compared to the next-to-leading order QCD prediction, with and without the inclusion of nonperturbative effects, in $\gamma N$ collisions at $\left\langle E_{\gamma}\right\rangle=145 \mathrm{GeV}$.

$\left\langle k_{T}^{2}\right\rangle$, and it can accommodate any value between 0.5 and $2 \mathrm{GeV}^{2}$.

\subsection{Bottom Production at Hadron Colliders}

The status of $b$ production at hadron colliders has been quite puzzling for some time. Owing to recent progress, however, the situation has considerably clarified. Let us briefly review the history of the theoretical predictions and experimental results on the $b$ spectrum at hadron colliders. Early calculations agreed quite well with UA1 measurements [44]. In fig. 16 the comparison between UA1 data and the theoretical calculation is displayed. As first pointed out in ref. [1], the perturbative computation of the $b$ cross section at hadron colliders reaches a difficult kinematical regime when

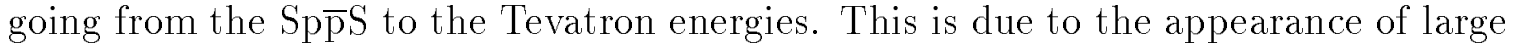
logarithms of $S / m^{2}$ (where $S$ is the CM energy squared, and $m$ is the mass of the heavy quark being produced) in the perturbative expansion (these are often referred to as small- $x$ effects). In other words, at very large $S$ the expansion parameter becomes $\alpha_{S} \log \left(S / m^{2}\right)$, which, for $S$ large enough, is of order 1 , thereby spoiling the 


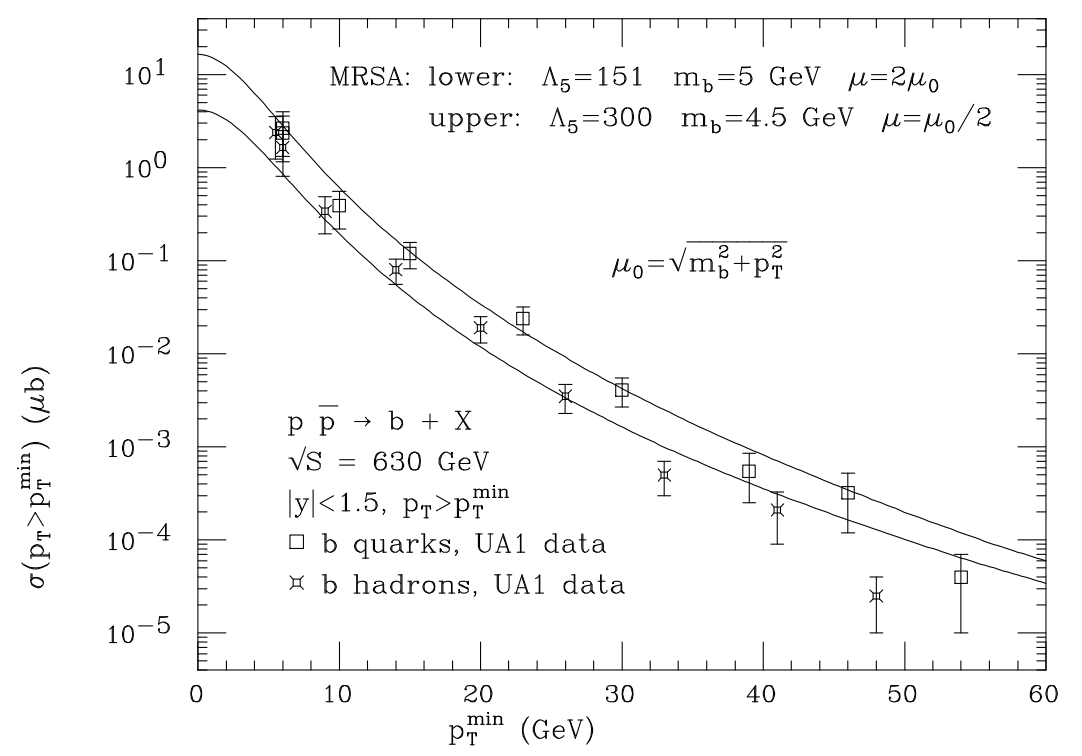

Figure 16: Bottom cross sections at UA1 versus theoretical predictions.

convergence of the perturbative expansion. The first predictions for $b$ cross sections at the Tevatron [1] were therefore given with lots of caveats. In addition to uncertainties in the gluon distribution at such small values of $x$, the perturbative expansion did not seem well-behaved, and it was believed that the resummation of all powers of $\alpha_{S} \log \left(S / m^{2}\right)$ was a priori necessary in order to get sensible results. The fact that UA1 results agreed well with theoretical predictions, while the Tevatron results [45] were much higher, could then be interpreted as an indication of small- $x$ effects. In the meantime, quite substantial theoretical work was performed on the small- $x$ problem [46]. In particular, in ref. [47] it was found that the resummation of small- $x$ effects increases the total rate by at most $30 \%$. This increased confidence in the original $\mathcal{O}\left(\alpha_{S}^{3}\right)$ computations $\left(\mathcal{O}\left(\alpha_{S}^{4}\right)\right.$ corrections are expected to be of that order of magnitude). However, since the early Tevatron results were 2 to 3 times higher than the theoretical predictions, this was not enough to explain the discrepancy. Independent efforts to modify the gluon distribution to reproduce the Tevatron results [48] came short of a fully satisfactory solution to the problem.

Recent experimental progress in $b$-production studies at the Tevatron detectors CDF [49] and D0 [50], has considerably changed the situation. First of all, a direct 
measurement of the inclusive $b \rightarrow J / \psi$ and $b \rightarrow \psi^{\prime}$ decays has become possible thanks to the detection of the secondary vertex of $b$ decays from which $\psi$ mesons originate [49]. This measurement has shown that the fraction of $\psi$ mesons coming from $b$ decays is significantly smaller than previously expected. As a result, the $b$-production cross section extracted from this measurement has decreased. Increased statistics has also significantly improved the observation of fully reconstructed $B \rightarrow \psi K^{(*)}$ decays, leading to an unambiguous measurement of the $B$-meson $p_{T}$ spectrum. In addition, the new D0 detector has recently presented its first measurement of the $b$ $p_{T}$ spectrum using inclusive semileptonic $b \rightarrow \mu X$ decays [50]. Finally, a measurement of double-differential $b$ production has been performed by CDF [51].

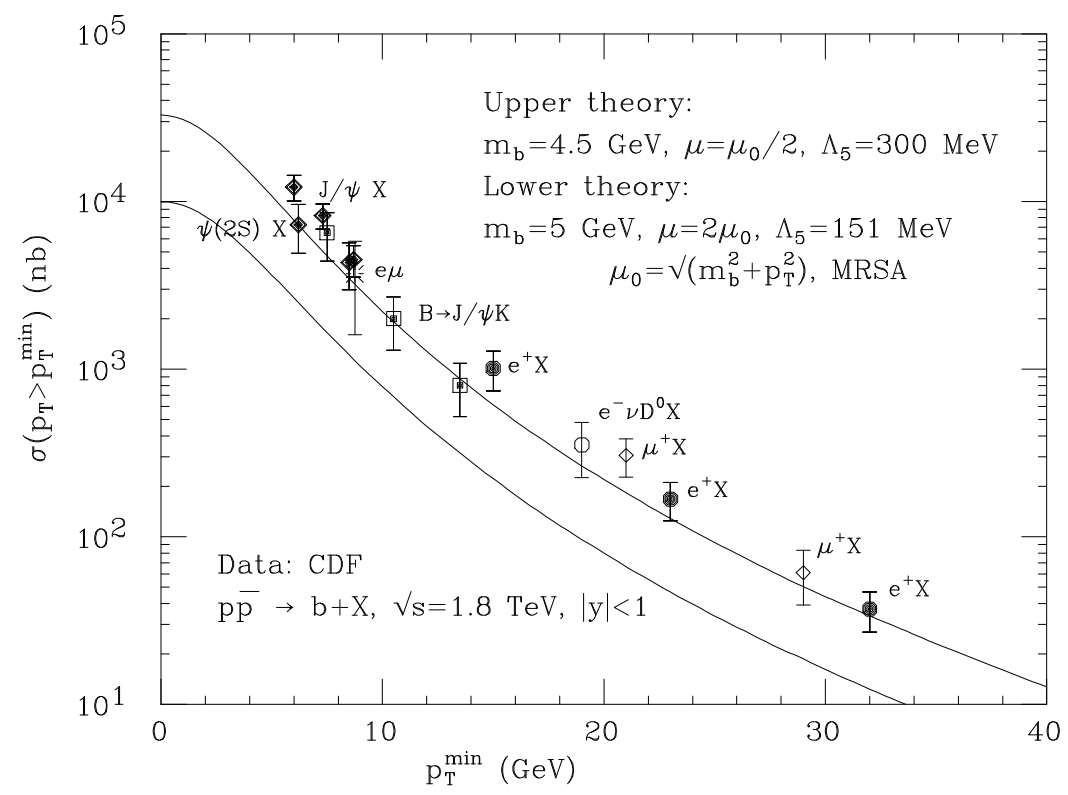

Figure 17: Bottom cross sections at CDF versus theoretical predictions.

The whole situation is summarized in figs. 17, 18 and 19, showing a comparison of the theoretical expectations with the results from CDF and D0 for integrated $p_{T}$ distributions of $b$ quarks and differential $p_{T}$ distributions of $B$ mesons. In the case of CDF we removed from the plot the old measurements relative to the $\psi$ decay modes, which have been superseded by the most recent results mentioned above.

The theoretical curves require some explanation. First of all, they do not differ 
much from the original prediction of ref. [1] using the DFLM structure functions ${ }^{4}$. New structure function fits, including the first results from HERA, have recently become available. We use in our prediction one of these sets, namely MRSA [52]. Since the values of $x$ probed by $b$ production at the Tevatron in the currently measured $p_{T}$ range only cover the region $x>5 \times 10^{-3}$, we observe no significant change relative to the results obtained using older fits.

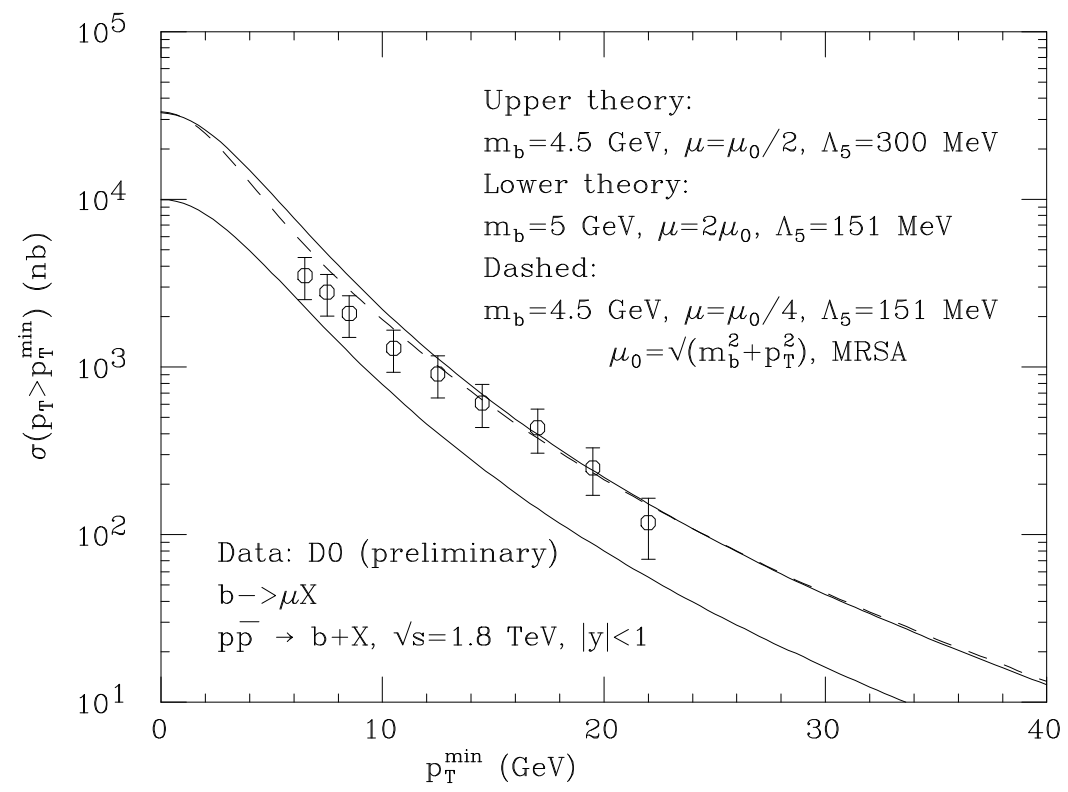

Figure 18: Bottom cross sections at D0 versus theoretical predictions.

The second important point is the choice of a range for $\Lambda_{5}$. Deep inelastic scattering results tend to favour smaller values of $\Lambda$. For example, the set MRSA uses $\Lambda_{5}=151 \mathrm{MeV}$. On the other hand, LEP data favour a higher value: the central value of $\Lambda_{5}$ at LEP is around $300 \mathrm{MeV}$. This value is also supported by other lower-energy results, such as the $\tau$ hadronic width (for a review of $\Lambda_{5}$ determinations, see ref. [28]). It is therefore sensible to use the range from 151 to $300 \mathrm{MeV}$ for $\Lambda_{5}$.

The upper curves in fig. 17 and fig. 18 correspond to the PDF set MRSA [52], $\Lambda_{5}=300 \mathrm{MeV}, m_{b}=4.5 \mathrm{GeV}$ and $\mu_{R}=\mu_{F}=\sqrt{p_{T}^{2}+m_{b}^{2}} / 2$. The lower curves

\footnotetext{
${ }^{4}$ In ref. [1] the scale was chosen in a slightly different way: $\mu_{0}=\sqrt{m_{b}^{2}+p_{T}^{\min 2}}$, which makes a $10 \%$ difference in the cross sections.
} 


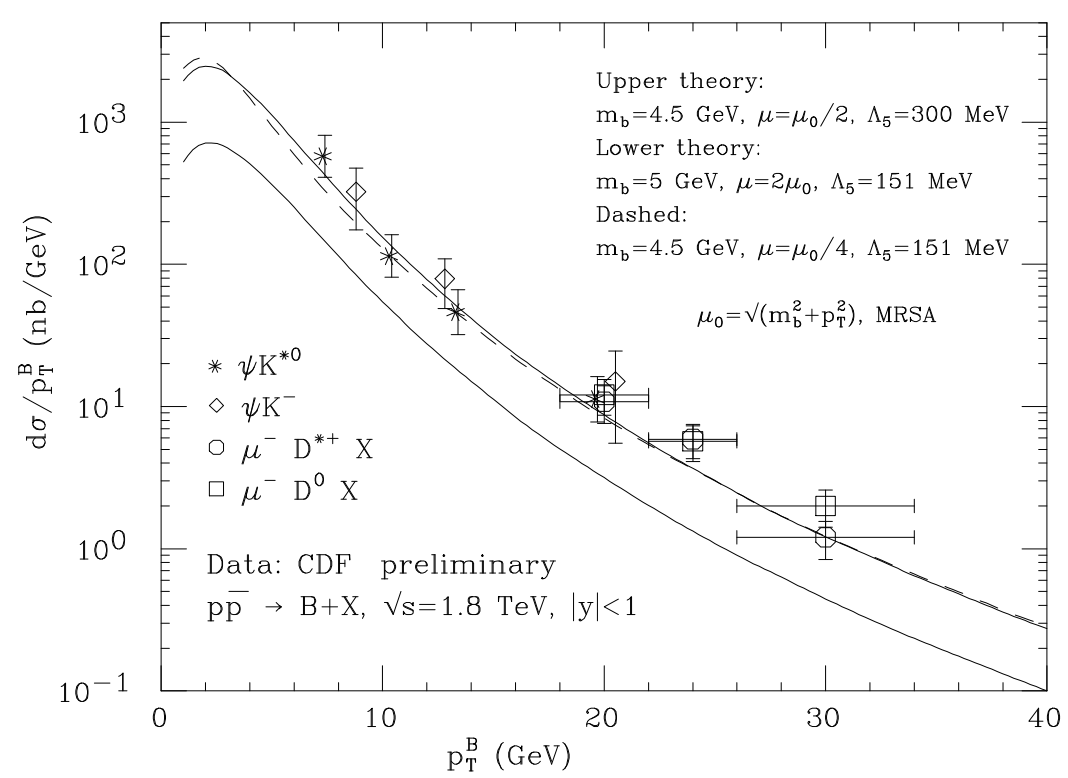

Figure 19: B-mesons differential $p_{T}$ distribution at $C D F$ versus theoretical predictions. Data and theory refer to a single charge state $\left(B^{0}\right.$ or $\left.B^{+}\right)$; the theory curves assume equal fractions of neutral and charged $B$ mesons from b-quark hadronization, with $f\left(B^{0}\right)=f\left(B^{ \pm}\right)=37.5 \%$.

correspond to $\Lambda_{5}=151 \mathrm{MeV}, m_{b}=5 \mathrm{GeV}$ and $\mu_{R}=\mu_{F}=2 \sqrt{p_{T}^{2}+m_{b}^{2}}$. As in previous sections, in the absence of fits with $\Lambda_{5}$ frozen to the desired values we chose to simply change the value of $\Lambda_{5}$ in the partonic cross section. A priori, one would expect that this amounts to an overestimate of the variation due to the uncertainty in $\Lambda_{5}$. As a test, we used the structure-function fits performed by the CTEQ collaboration [29], which presents results both for the best DIS fit of $\Lambda_{5}\left(\Lambda_{5}=152 \mathrm{MeV}\right.$, set CTEQ1M) and for a value artificially frozen to a number closer to the LEP measurement (set CTEQ1ML, $\Lambda_{5}=220 \mathrm{MeV}$ ). In fig. 20 we plot the ratio of the $b$ cross sections obtained using the two sets CTEQ1M and CTEQ1ML, together with the ratio between the result of the default MRSA set and of MRSA with $\Lambda_{5}$ fixed at $220 \mathrm{MeV}$. We used $m_{b}=4.75 \mathrm{GeV}$ and $\mu_{R}=\mu_{F}=\sqrt{p_{T}^{2}+m_{b}^{2}}$. As is clear from the figure, the two results are equal to within a few per cent, indicating that varying $\Lambda_{5}$ within a limited range, without refitting the parton distributions, is a reasonable way to estimate the systematic effect of the $\Lambda_{5}$ uncertainty on the $b$ cross section. 
In fig. 18 we also show as a dashed line the theoretical prediction obtained using a rather extreme choice of scale, namely $\mu_{R}=\mu_{F}=\mu_{0} / 4$, toghether with $m_{b}=4.5 \mathrm{GeV}$ and MRSA PDF $\left(\Lambda_{5}=151 \mathrm{MeV}\right)$. It is interesting to notice that this choice results in rates almost equal to those obtained using $\mu_{R}=\mu_{F}=\mu_{0} / 2$ and the extreme value of $\Lambda_{5}=300 \mathrm{MeV}$. This suggests that indeed most of the variation in the cross section is simply due to the changes in $\alpha_{S}$, as $\alpha_{S}=\alpha_{S}\left(\mu_{R} / \Lambda\right)$; the argument is the same for the two choices $\mu_{R}=\mu_{0} / 2, \Lambda_{5}=300 \mathrm{MeV}$, and $\mu_{R}=\mu_{0} / 4, \Lambda_{5}=151 \mathrm{MeV}$.

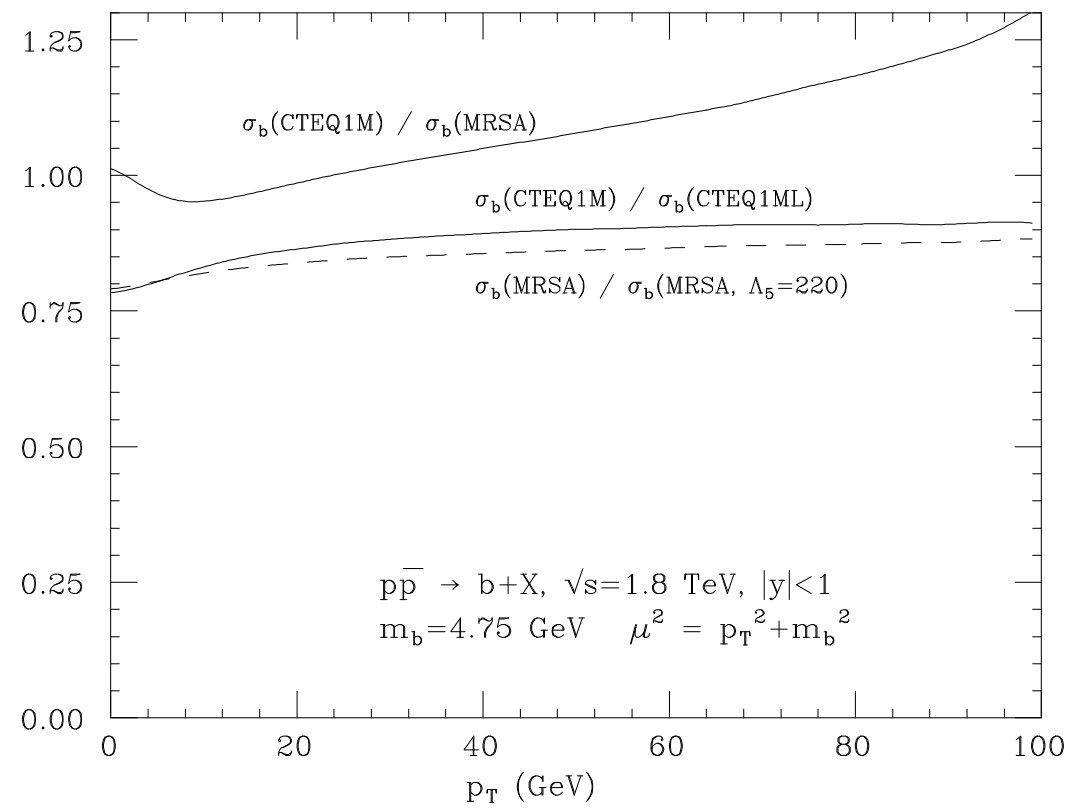

Figure 20: Dependence of predicted bottom cross section on different choices of structure functions and values of $\Lambda_{5}$. See the text for a complete discussion.

As for the $B$-meson differential $p_{T}$ distribution, shown in fig. 19, we convoluted the $b$ quark theoretical curve with a Peterson fragmentation function [43], using $\epsilon=0.006$ [53], and we rescaled the curves by a constant factor of $37.5 \%$, to account for the expected fraction of $B$ mesons of a given charge. Observe that we did not apply a fragmentation function to our predictions in figs. 16, 17 and 18 since in these cases the experimental collaborations present results for bare heavy quarks.

For completeness, we include in fig. 20 the ratio between the predictions obtained using the two default sets, CTEQ1M and MRSA. This number is equal to 1 to within $5 \%$ for a large range of $p_{T}$. While such a stability is partly artificial, being related to 
the large overlap of correlated measurements entering the determination of the parton distribution fits, it however suggests that by now the uncertainty in the structure functions does not leave much room by itself for significant changes in the expected $b$ cross section at Tevatron energies.

Coming back to the comparison of theory and data, from figs. 17 and 19 we see that the CDF data points are now consistent with the fixed-order theoretical prediction, although on the high side. The D0 points, instead, comfortably sit within the theoretical range. Once the experimental statistics and systematics will be further reduced, it will be reasonable to assume that residual discrepancies of the same order as those currently observed may be explained in terms of small- $x$ effects. Additional theoretical studies of these effects, such as a better understanding of the matching with the fixed-order next-to-leading-order calculations, should therefore be pursued.

\section{Double-differential distributions}

Correlations between charmed particles in hadro- and photoproduction have been studied by many experiments (see for example refs. [16], [41] and [54-57]), which reported distributions of the azimuthal distance between the charmed hadrons, the rapidity difference, the invariant mass and the $p_{T}$ of the pair. In what follows we will focus on the distributions of $\Delta \phi$, defined as the angle between the projections of the momenta of the pair onto the transverse plane, and of the transverse momentum of the pair. Leading-order QCD predicts that the heavy-quark pair will be produced exactly in the back-to-back configuration, corresponding to $\Delta \phi=\pi$ and $p_{T}(Q \bar{Q})=0$. Nextto-leading-order corrections, as well as nonperturbative effects, can cause a broadening of these distributions, as illustrated in refs. [12] and [10]. In the hadroproduction case, the data show some enhancement of the $\Delta \phi$ distribution around $\pi$. There is however no sound agreement on the significance of the enhancement observed by different experiments. For example, the WA75 collaboration [41] favours a relatively flat distribution. The E653 collaboration [55] has mild evidence for a peak in the $\Delta \phi=\pi$ bin. A recent analysis performed by the WA92 collaboration [58] shows clear evidence of a back-to-back enhancement of the distribution.

We addressed the question if next-to-leading-order QCD predictions can account for the available experimental data. We have chosen, as an illustration, the cases of 
the WA75 and the WA92 results, which have both been obtained in $\pi^{-} N$ collisions at the same energy, $E_{b}=350 \mathrm{GeV}$. In figs. 21 and 22 we show the next-to-leading result superimposed to the data of the WA75 and WA92 experiments, respectively. In both cases, we see that the experimental data are broader than the theoretical

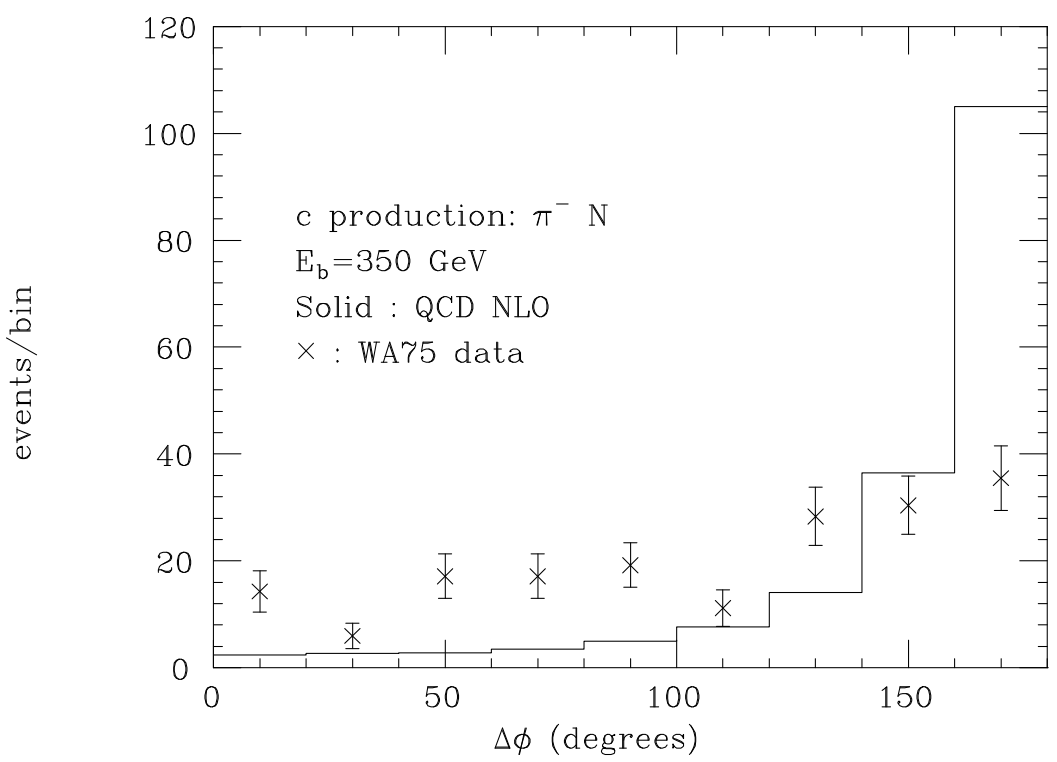

Figure 21: Azimuthal correlation in charm production: NLO calculation versus the WA75 data.

curves.

One should however take into account also nonperturbative effects, as in the case of single-inclusive distributions. We have computed the $\Delta \phi$ distribution in perturbative QCD with an intrinsic transverse momentum of the incoming partons as described in subsection 3.1. We observe that the use of a fragmentation function has no effect on the $\Delta \phi$ distribution, since it does not affect momentum directions. The leading-order prediction supplemented by the transverse primordial momentum of the partons yields already a rather broad distribution [12]. The next-to-leading-order prediction, when supplemented with the effect of the primordial transverse momentum with $\left\langle k_{T}^{2}\right\rangle=0.5$ $\mathrm{GeV}^{2}$, gives a rather consistent description of the heavy flavour azimuthal correlation in the case of the WA92 data, as shown in fig. 23 . A value of $\left\langle k_{T}^{2}\right\rangle=0.5 \mathrm{GeV}^{2}$ is very plausible. It is however impossible to describe the WA75 data in the same way. In 


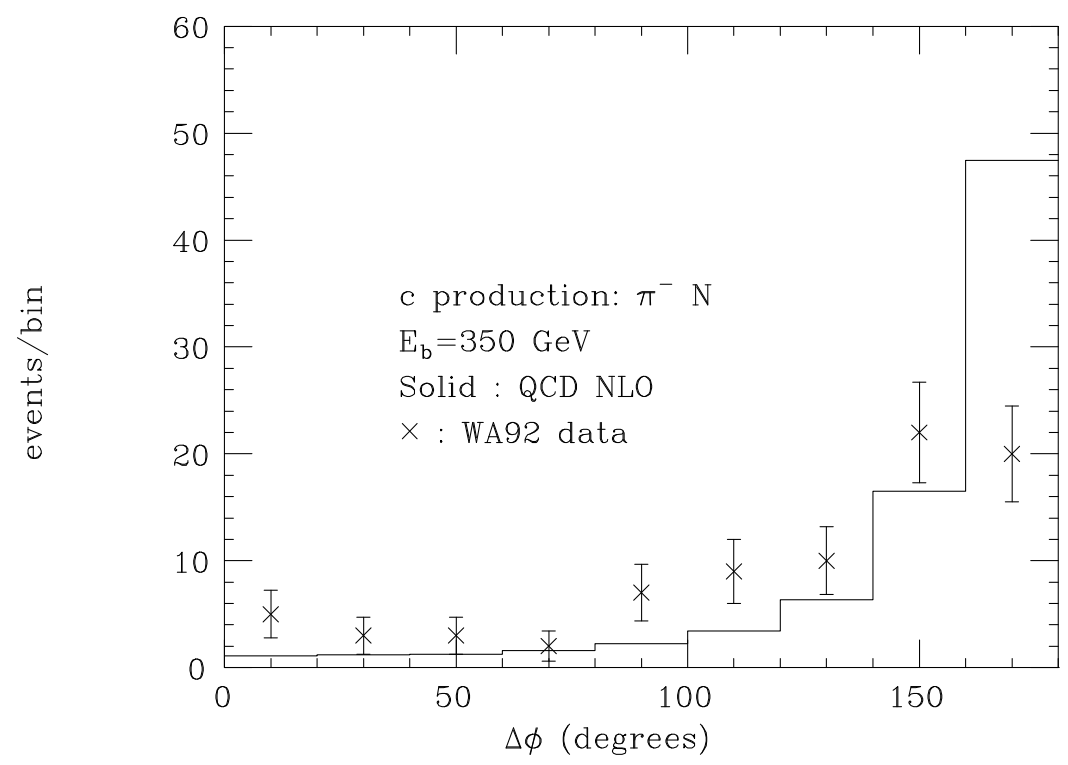

Figure 22: Azimuthal correlation in charm production: NLO calculation versus the WA92 data.

fig. 24 we show the comparison of the next-to-leading order result supplemented with a primordial transverse momentum effect with $\left\langle k_{T}^{2}\right\rangle=0.5 \mathrm{GeV}^{2}$ and $1 \mathrm{GeV}^{2}$.

The WA75 collaboration also published in ref. [41] the distribution of the transverse momentum of the heavy-quark pair. This distribution is very hard, and in this case the theoretical prediction supplemented with a parton primordial transverse momentum with $\left\langle k_{T}^{2}\right\rangle=1 \mathrm{GeV}^{2}$ is insufficient to reproduce the data, as displayed in fig. 25. Unlike the azimuthal correlation, the pair transverse momentum distribution is affected by fragmentation effects, since these effects can randomly degrade the momenta of the quark and antiquark by different amounts. Fragmentation effects, however, also moderate the pair transverse momentum arising from gluon radiation or from an intrinsic parton transverse momentum. We have verified that at the end, at $E_{b}=350 \mathrm{GeV}$, the fragmentation effect always tends to soften the pair transverse momentum distribution.

Summarizing, the WA75 data require a very large intrinsic transverse momentum of the incoming parton both for the azimuthal correlation and for the pair transverse momentum. On the other hand, the $\Delta \phi$ distribution measured by WA92 is consis- 


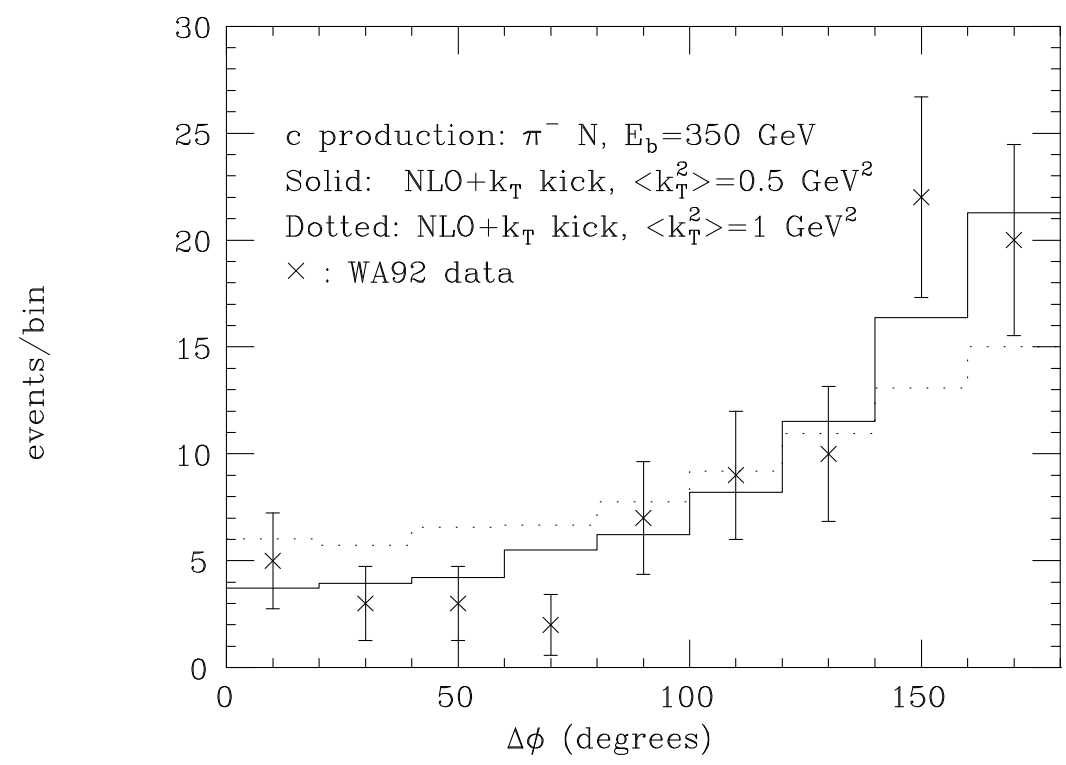

Figure 23: Next-to-leading-order QCD result supplemented with a primordial transverse momentum for the incoming partons, compared with the WA92 data.

tent with a reasonably moderate intrinsic $k_{T}$ distribution. Clarification of this issue requires more experimental information.

We observe that the procedure of adding an intrinsic transverse momentum to the incoming partons in the perturbative computation is not theoretically well defined. In fact, the perturbative expansion itself does provide, via gluon emission, a transverse momentum to the partons that enter the hard subprocess. We find for example that changing the renormalization scale $\mu_{R}$ to lower values leads to a broader $\Delta \phi$ distribution. This is illustrated in fig. 26 , where we see that for a choice of $\mu_{R}=\mu_{0} / 2$ there is no need for an intrinsic transverse momentum in order to reproduce the WA92 data. As an additional remark, we mention that in ref. [12] we verified, using the Monte Carlo HERWIG, that perturbative higher-order effects do not significantly affect the shape of the $\Delta \phi$ distributions.

Photoproduction of heavy quarks [57] is another example in which a $k_{T}$ kick would induce broader correlations. In fig. 27 the azimuthal correlation measured by the E687 collaboration is given, together with the next-to-leading-order result. The next- 


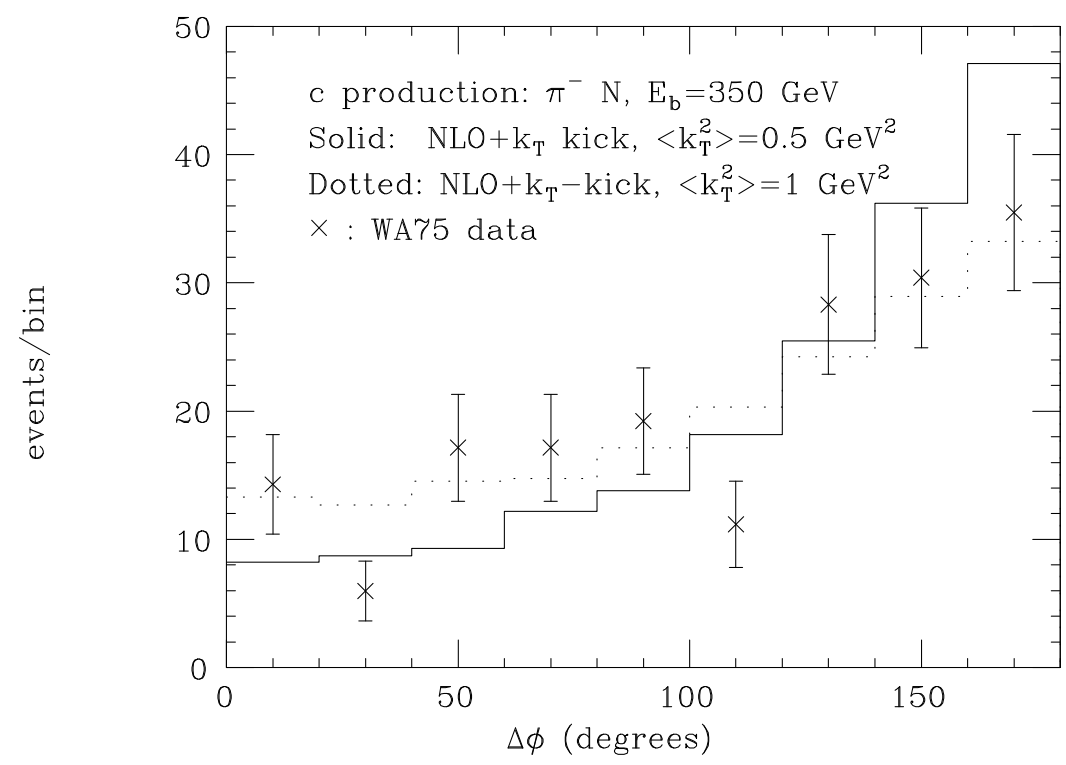

Figure 24: Next-to-leading-order QCD result supplemented with a primordial transverse momentum for the incoming partons, compared with the WA75 data.

to-leading-order result supplemented by an intrinsic $k_{T}$ of the incoming partons is also shown, for $\left\langle k_{T}^{2}\right\rangle=0.5 \mathrm{GeV}^{2}$ and $\left\langle k_{T}^{2}\right\rangle=1 \mathrm{GeV}^{2}$. As one can see, the data do not require a large intrinsic transverse momentum. All curves give a reasonable representation of the data, the one with $\left\langle k_{T}^{2}\right\rangle=0.5 \mathrm{GeV}^{2}$ being slightly better. A similar conclusion applies to the NA14/2 data (which are however affected by larger uncertainties), as shown in fig. 28. Another distribution, which is very sensitive to the intrinsic transverse momentum of the partons, is the transverse momentum of the heavy-quark pair, displayed in fig. 29. In this case, we see that the data favour $\left\langle k_{T}^{2}\right\rangle=1 \mathrm{GeV}^{2}$.

New interesting results have appeared recently on the azimuthal correlations of $b \bar{b}$ pairs in high-energy hadron collisions, both by the UA1 experiment [59] and by the CDF experiment [51]. The results are in very good agreement with the NLO calculations of ref. [3]. Here the mass of the heavy quarks and their energies in the samples used for the measurement are large enough for no significant contribution to be expected to originate from the intrinsic $k_{T}$ of the initial partons. Additional higherorder perturbative corrections coming from either small- $x$ effects [60] or multiple soft- 


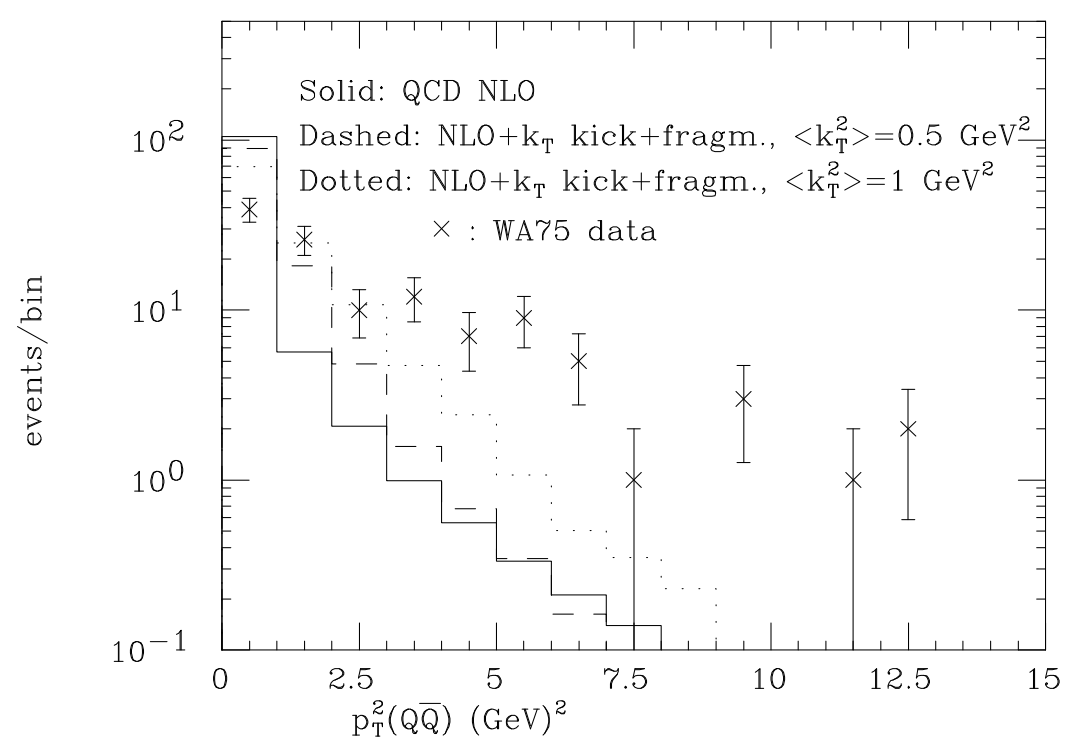

Figure 25: Next-to-leading-order $Q C D$ result for the $p_{T}^{2}(Q \bar{Q})$ supplemented with a primordial transverse momentum for the incoming partons, compared with the WA75 data.

gluon emission [5] could in principle modify the shape of the azimuthal correlations at these energies. Future data with better statistics and over a wider range of momenta will make more refined comparisons possible.

\section{Conclusions}

We have presented a comparison between charm and bottom production data at fixed-target and collider experiments, and theoretical predictions. All the totalcross-section data are in reasonable agreement with the QCD next-to-leading-order predictions, once the large theoretical uncertainties are properly taken into account, with the exception of the results of ref. [30], which are very difficult to explain in the context of perturbative QCD. The measurements for charm production in pionnucleon, proton-nucleon and photon-nucleon collisions are consistent with a quark mass value of $1.5 \mathrm{GeV}$. The data for bottom production in pion-nucleon collisions are consistent with the usually assumed range for the bottom mass $4.5<m_{b}<5 \mathrm{GeV}$. 


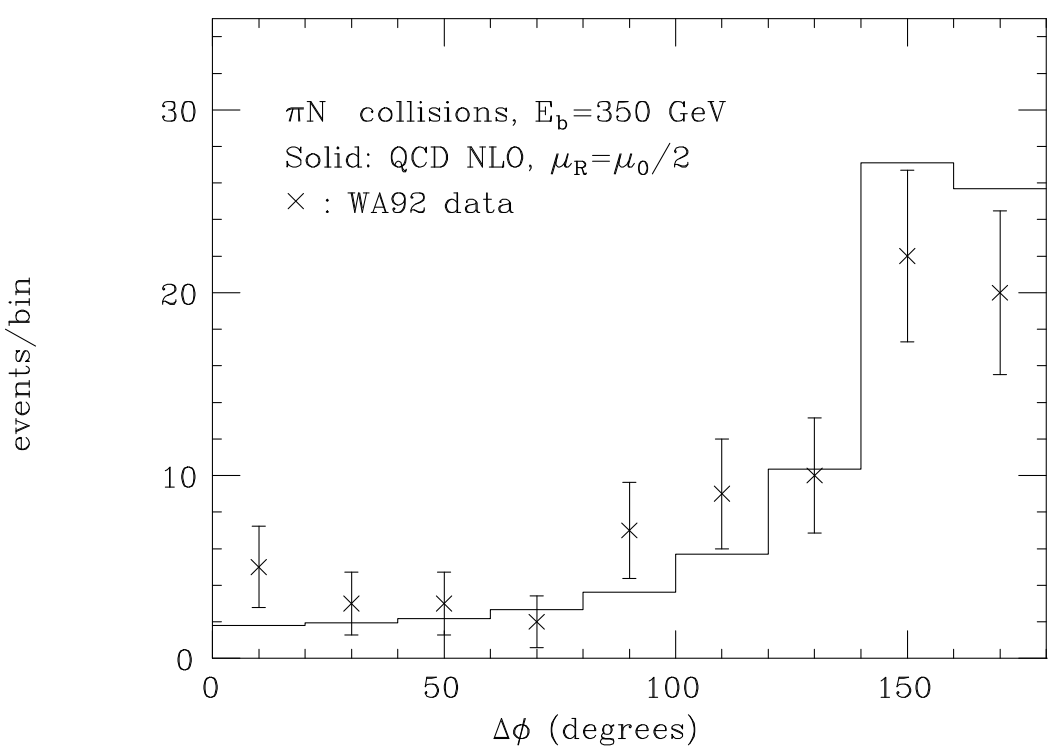

Figure 26: Next-to-leading-order QCD result calculated with $\mu_{R}=\mu_{0} / 2$ compared with the WA92 data.

We have shown that, in the case of charm, the pure QCD perturbative results are not adequate to describe the observed distributions, and that the inclusion of nonperturbative effects is necessary. We attempted to model these effects by giving a randomly distributed transverse momentum $\left(k_{T}-\mathrm{kick}\right)$ to the partons entering the hard subprocesses, and by applying to the final-state heavy quarks a Peterson fragmentation function in order to model hadronization.

For the single-inclusive transverse momentum distribution, the agreement with the photoproduction data is quite satisfactory for values of $\left\langle k_{T}^{2}\right\rangle$ between 0.5 and 2 $\mathrm{GeV}^{2}$. These distributions are not very sensitive to the value of $\left\langle k_{T}^{2}\right\rangle$. They do however show a slight preference for large values of $\left\langle k_{T}^{2}\right\rangle$, at least for $m_{c}=1.5 \mathrm{GeV}$. In the hadroproduction case we were able to perform a meaningful comparison only for the data of the WA82 and E769 collaboration, for reasons explained in subsection 3.1. We get a good agreement with the WA82 data for $\left\langle k_{T}^{2}\right\rangle$ around $2 \mathrm{GeV}^{2}$.

We have also shown that the measured $x_{F}$ distribution can be reproduced by the perturbative QCD next-to-leading-order result only for the non-leading $D$-meson hadroproduction. Due to the failure of the factorization theorem in the high- $x_{F}$ region, 


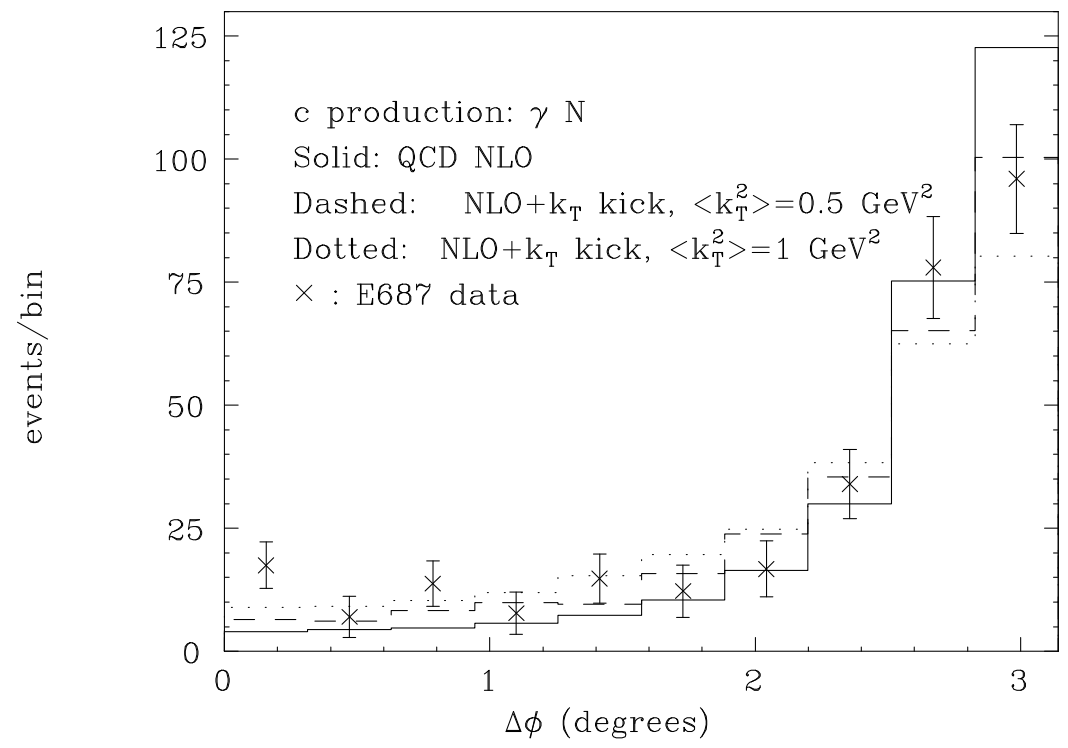

Figure 27: Azimuthal correlation of $D \bar{D}$ pair versus the perturbative result in photoproduction for the E687 experiment.

we were not able to supplement our perturbative calculation with a description of the hadronization phenomena. We remind the reader that in ref. [12] this problem was studied using a parton shower approach, which led to harder distributions, possibly consistent with the data.

The new measurements of the transverse momentum of $b$ hadrons, recently performed by the CDF and D0 collaborations, have been compared to theoretical predictions. These data appear now to be consistent with the pure QCD result, although they are on the high side of the prediction band. The $b$-hadron distribution is satisfactorily described by convoluting the perturbative result with the Peterson fragmentation function. We verified that the addition of a $k_{T}$-kick to the incoming partons has no sizeable effect, and that the results obtained are quite stable with respect to the choice of the parametrization of the structure functions.

We also compared theoretical predictions with experimental measurements in the case of double-differential distributions. We considered the distribution in the transverse momentum of the heavy-quark pair, and the distribution in the azimuthal distance between the two heavy quarks. For the transverse momentum of the heavy- 


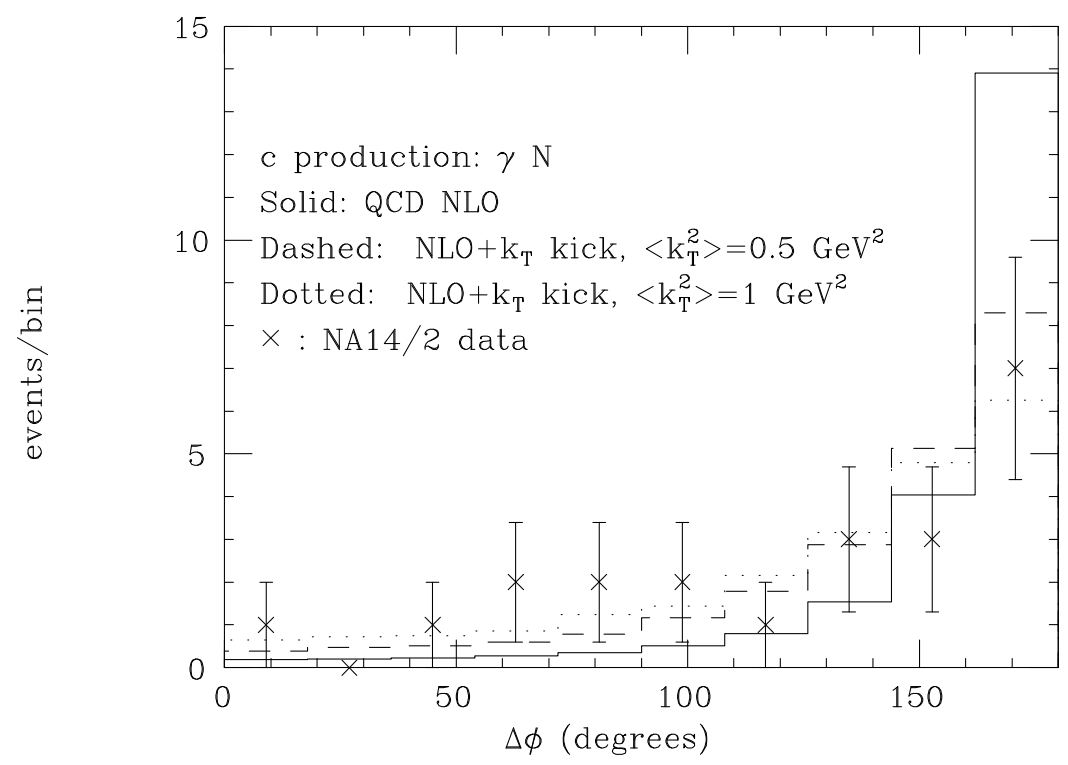

Figure 28: Azimuthal correlation of $D \bar{D}$ pair versus the perturbative result in photoproduction for the NA14/2 experiment.

quark pair, we find that a value of $\left\langle k_{T}^{2}\right\rangle=1 \mathrm{GeV}^{2}$ fits the photoproduction data of the E687 experiment well, while in the hadroproduction case the experimental distribution (measured by the WA75 experiment) is much harder than the theoretical one for any plausible value of $\left\langle k_{T}^{2}\right\rangle$. For the $\Delta \phi$ distribution we found that in almost all cases a definite choice of $\left\langle k_{T}^{2}\right\rangle$ is sufficient to reproduce the data. However, different data sets favour different values of $\left\langle k_{T}^{2}\right\rangle$, ranging from $0.5 \mathrm{GeV}^{2}$ for the WA92 experiment to $1 \mathrm{GeV}^{2}$ for WA75. Photoproduction data are well described by both values.

We did not discuss the azimuthal distance of $b$ pairs at collider experiments, since these analyses were performed by the UA1 and CDF collaborations, using the theoretical results of ref. [3]. Excellent agreement with the QCD distributions was found in both cases.

We did not attempt to include in this work a full study of the mass dependence of the distributions we calculated. In fact, since some data sets are not quite consistent with one another, this is premature at this stage. The tools developed in the present work for the inclusion of the primordial transverse momentum and for the inclusion of the fragmentation function in the next-to-leading-order calculation will be made 


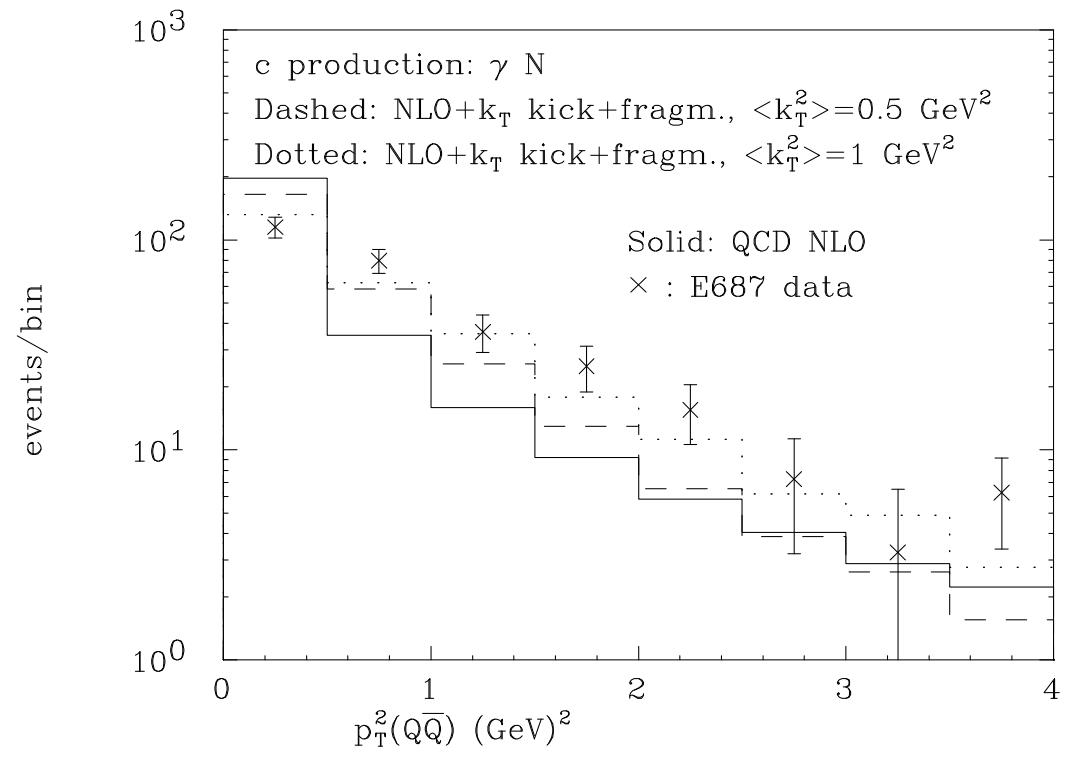

Figure 29: Transverse momentum distribution of the $D \bar{D}$ pair versus the perturbative result for the E687 experiment.

available upon request in the form of computer programs, so that the experimental collaborations will be able to perform such studies as more data become available.

\section{Acknowledgements}

The work of MLM is supported in part by the EEC Programme "Human Capital and Mobility", Network "Physics at High Energy Colliders", contract CHRX-CT930537 (DG 12 COMA).

We would like to thank F. Antinori, J. Appel, K. Bazizi, G. Bellini, A. Cardini, G. Catanesi, R. Gardner, P. Karchin, V. Papadimitriou, S. Passaggio, B. Osculati, M. Purhoit, C. Roda, L. Rossi, M. Schub, T. Sjöstrand, P. Sphicas, S. Tkaczyk and J. Wilcox for useful discussions. 


\section{References}

[1] P. Nason, S. Dawson and R. K. Ellis, Nucl. Phys. B303(1988)607; B327(1988)49.

[2] W. Beenakker et al., Nucl. Phys. 303(1991)507; Phys. Rev. D40(1989)54.

[3] M.L. Mangano, P. Nason and G. Ridolfi, Nucl. Phys. B373(1992)295.

[4] E. Laenen, J. Smith and W.L. van Neerven, Nucl. Phys. B369(1992)543.

[5] E. Berger and R. Meng, Phys. Rev. D49(1994)3248.

[6] M. Cacciari and M. Greco, preprint FNT/T-93/43 (University of Pavia), hepph/9311260 (1993).

[7] R.K. Ellis and P. Nason, Nucl. Phys. B312(1989)551.

[8] J. Smith and W.L. Van Neerven, Nucl. Phys. B374(1992)36.

[9] E. Laenen, S. Riemersma, J. Smith and W.L. Van Neerven, Nucl. Phys. B392(1993)162 and 229.

[10] S. Frixione, M.L. Mangano, P. Nason and G. Ridolfi, Nucl. Phys. B412(1994)225.

[11] G. Altarelli, M. Diemoz, G. Martinelli and P. Nason, Nucl. Phys. B308(1988)724; R.K. Ellis, Phys. Lett. B259(1991)492;

E. Berger, R. Meng and W.K. Tung, Phys. Rev. D46(1992)1895.

[12] M. Mangano, P. Nason and G. Ridolfi, Nucl. Phys. B405(1993)507.

[13] K. Kodama et al., E653 Coll., Phys. Lett. B263(1991)573.

[14] S. Barlag et al., ACCMOR Coll., Z. Phys. C39(1988)451.

[15] R. Ammar et al., LEBC-MPS Coll., Phys. Rev. Lett. 61(1988)2185.

[16] M. Aguilar-Benitez et al., LEBC-EHS Coll., Z. Phys. C40(1988)321.

[17] M. Aguilar-Benitez et al., LEBC-EHS Coll., Phys. Lett. B161(1985)400.

[18] S. Barlag et al., ACCMOR Coll., Z. Phys. C49(1991)555.

[19] K. Kodama et al., E653 Coll., Phys. Lett. B284(1992)461. 
[20] S. Aoki et al., WA75 Coll., Progr. Theor. Phys. 87(1992)1305.

[21] M. P. Alvarez et al., NA14/2 Coll., Z. Phys. C60(1993)53.

[22] M. J. Leitch et al., E789 Coll., Phys. Rev. Lett. 72(1994)2542.

[23] M. Schub, private communication.

[24] G. Marchesini and B.R. Webber, Nucl. Phys. B310(1988)461.

[25] P. Harriman, A. Martin, R. Roberts and J. Stirling, Phys. Rev. D37(1990)798.

[26] P.J.Sutton, A.D. Martin, R.G. Roberts and W.J. Stirling, Phys. Rev. D45(1992)2349.

[27] A.D. Martin, R.G. Roberts and W.J. Stirling, Phys. Rev. D43(1991)3648.

[28] S. Catani, preprint DFF 194/11/93, to appear in the Proceedings of the EPS conference, Marseille, 1993.

[29] J. Botts et al., Phys. Lett. B304(1993)159.

[30] G. Bari et al., Nuovo Cimento 104A(1991)1787;

M. Basile et al., Nuovo Cimento 65A(1981)391.

[31] J.P. Albanese et al., WA75 Coll., Phys. Lett. 108B(1982)361.

[32] M.G. Catanesi et al., WA78 Coll, Phys. Lett. 231B(1989)328.

[33] P. Bordalo et al., NA10 Coll., Z. Phys. C39(1988)7.

[34] K. Kodama et al., E653 Coll., Phys. Lett. B303(1993)359.

[35] R. Jesik et al., E672-E706 Coll., preprint Fermilab PUB-94/095-E.

[36] S.P.K. Tavernier, Rep. Progr. Phys. 50(1987)1439.

[37] J.C. Anjos et al., E691 Coll., Phys. Rev. Lett. 62(1989)513; Phys. Rev. Lett. $65(1990) 2503$.

[38] G. Bellini, Proceedings of Les Rencontres de Physique de la Vallée d'Aoste, La Thuile, March 1994.

[39] M.I. Adamovich et al., WA82 Coll., Nucl. Phys. (Proc. Suppl.) B27(1992)212. 
[40] J.A. Alves et al., E769 Coll., Phys. Rev. Lett. 69(1992)3147.

[41] S. Aoki et al., WA75 Coll., Progr. Theor. Phys. 87(1992)1315; Phys. Lett. B209(1988)113.

[42] B. L. Combridge, Nucl. Phys. B151(1979)429;

S. J. Brodsky et al., Phys. Lett. B93(1980)451.

[43] C. Peterson, D. Schlatter, I. Schmitt and P. Zerwas, Phys. Rev. D27(1983)105.

[44] C. Albajar et al., UA1 Coll., Phys. Lett. B256(1991)121.

[45] F. Abe et al., CDF Coll., Phys. Rev. Lett. 68(1992)3403; 69(1992)3704; 71(1993)500, 2396 and 2537.

[46] R.K. Ellis and D.A. Ross, Nucl. Phys. B345(1990)79;

S. Catani, M. Ciafaloni and F. Hautmann, Phys. Lett. B242(1990)97, Nucl. Phys. B366(1991)135, Nucl. Phys. (Proc. Suppl.) 23B(1991)328.

[47] J.C. Collins and R.K. Ellis, Nucl. Phys. B360(1991)3.

[48] E. Berger, R. Meng and W.K. Tung, Phys. Rev. D46(1992)1895.

[49] F. Abe et al., CDF Coll., Fermilab-Conf-94/134-E; Fermilab-Conf-94/136-E; Fermilab-Conf-94/141-E.

[50] K. Bazizi, D0 Coll., to appear in the Proceedings of the XXIX Rencontres de Moriond, Méribel, France, March 1994.

[51] F. Abe et al., CDF Coll., Fermilab-Pub-94/131-e, submitted to Phys. Rev. Lett.; Fermilab-Conf-94/129-E.

[52] A.D. Martin, W.J. Stirling and R.G. Roberts, Rutherford Lab preprint RAL94-055, DTP/94/34 (1994).

[53] J. Chrin, Z. Phys. C36(1987)163.

[54] S. Barlag et al., ACCMOR Coll., Phys. Lett. B257(1991)519.

[55] K. Kodama et al., E653 Coll., Phys. Lett. B263(1991)579.

[56] M. Aguilar-Benitez et al., LEBC-EHS Coll., Phys. Lett. B164(1985)404. 
[57] M.P. Alvarez et al., NA14/2 Coll., Phys. Lett. B278(1992)385,

V. Arena et al., E687 Coll., Phys. Lett. B308(1993)194;

M.I. Adamovich et al., Photon Emulsion Coll., Phys. Lett. B187(1987)437 and references therein.

[58] A. Cardini, WA92 Coll., to appear in the Proceedings of the XXIX Rencontres de Moriond, Méribel, France, March 1994; Ph.D thesis, Pisa University, unpublished.

[59] C. Albajar et al., UA1 Coll., Z. Phys. C61(1994)41.

[60] M.L. Mangano, Z. Phys. C58(1993)651. 